\title{
1 Hey2 restricts cardiac progenitor addition to the developing heart
}

2

3 Natalie Gibb ${ }^{1}$, Savo Lazic ${ }^{1,3}$, Ashish R. Deshwar ${ }^{1,3}$, Xuefei Yuan ${ }^{1-3}$, Michael D. Wilson ${ }^{2,3}$ and

$4 \quad$ Ian C. Scott ${ }^{1,3-5 *}$

5

6 'Program in Developmental and Stem Cell Biology, The Hospital for Sick Children, Toronto,

7 Ontario, M5G 0A4, Canada

$8{ }^{2}$ Program in Genetics and Genome Biology, The Hospital for Sick Children, Toronto,

9 Ontario, M5G 0A4, Canada

$10{ }^{3}$ Department of Molecular Genetics, University of Toronto, Canada

$11{ }^{4}$ Ted Rogers Centre for Heart Research, Toronto, Canada

$12{ }^{5}$ Heart and Stroke Richard Lewar Centres of Excellence in Cardiovascular Research, Toronto,

13 Canada

14

$15 *$ Author for correspondence (ian.scott@ sickkids.ca) 


\section{ABSTRACT}

17 A key event in vertebrate heart development is the timely addition of second heart

18 field (SHF) progenitor cells to the poles of the heart tube. This accretion process

19 must occur to the proper extent to prevent a spectrum of congenital heart defects

20 (CHDs). However, the factors that regulate this critical process are poorly understood.

21 Here we demonstrate that Hey2, a bHLH transcriptional repressor, restricts SHF

22 progenitor accretion to the zebrafish heart. hey2 expression demarcated a distinct

23 domain within the cardiac progenitor population. In the absence of Hey2 function an

24 increase in myocardial cell number and SHF progenitors was observed. We found that

25 Hey2 limited proliferation of SHF-derived cardiomyocytes in a cell-autonomous

26 manner, prior to heart tube formation, and further restricted the developmental

27 window over which SHF progenitors were deployed to the heart. Taken together, our

28 data suggests a role for Hey2 in controlling the proliferative capacity and cardiac

29 contribution of late-differentiating cardiac progenitors. 


\section{INTRODUCTION}

31 Cardiac development is regulated by the activity of concerted signaling, transcriptional and morphogenetic events. Subtle perturbations in these processes, either genetic or environmental, can lead to congenital heart defects (CHD), the most common class of congenital anomalies. It is now evident that the vertebrate heart is built from two populations of progenitor cells, termed the first heart field (FHF) and second heart field (SHF), which contribute to the heart in two successive windows of differentiation. Cells of the FHF differentiate in an initial wave of cardiogenesis, resulting in formation of the linear heart tube. Over a well-defined developmental window, multi-potent, late-differentiating progenitors of the SHF migrate into the poles of the heart tube to extensively remodel and add structure to the heart (Cai et al., 2003; Hutson et al., 2010; Kelly, 2012; van den Berg et al., 2009). It remains under debate however whether these populations of cardiac progenitor cells (CPCs) represent distinct populations with unique molecular signatures, or whether they exist as one population with a gradient in timing for deployment to the heart (Abu-Issa et al., 2004; Ivanovitch et al., 2017; Moorman et al., 2007).

In zebrafish, several fate-mapping studies have found that SHF progenitors give rise to the distal portion of the ventricular myocardium and smooth muscle of the outflow tract (OFT; (Guner-Ataman et al., 2013; Hami et al., 2011; Zeng and Yelon, 2014; Zhou et al., 2011). Embryological manipulations in chick and SHF-restricted mutation of CHD-associated genes in the mouse have firmly established that defects in SHF development are a major contributor to CHD (Cai et al., 2003; Prall et al., 2007; Ward et al., 2005). As a key driver of cardiac morphogenesis, the balance of proliferation, "stemness" and cardiac differentiation events in the SHF progenitor pool must be tightly regulated. Several signaling pathways have been implicated in the development of the SHF (Li et al., 2016; Mandal et al., 2017; Prall et al., 2007; Ryckebusch et al., 2008; Sirbu et al., 2008; Tirosh-Finkel et al., 2010; Zhao et al., 2014). Notable amongst these are fibroblast growth factor (FGF) and retinoic acid (RA) signaling. The permissive signals from RA towards FGF signaling create a mutual opposition for regulating the specification and differentiation of cardiac progenitor populations (Ilagan et al., 2006; Park et al., 2008; Rochais et al., 2009; 
63 (Isl1), Nkx2.5 and Fosl2 are essential for maintaining the SHF progenitor pool (Cai et

64 al., 2003; de Pater et al., 2009), yet the full make-up of the transcriptional network required to precisely regulate the behavior of the late-differentiating cardiac progenitor population remains unclear. subfamily, which act as transcriptional repressors during embryonic development (Davis and Turner, 2001). Zebrafish hey genes exhibit more restricted expression patterns compared to mammals, with hey 2 being the only family member detectably expressed in the heart (Winkler et al., 2003). Studies in mice have shown that a disruption in hey2 can lead to ventricular septal defects (VSD) as well as other CHDs and cardiomyopathy (Donovan et al., 2002; Sakata et al., 2002). Mutation in zebrafish hey2 leads to a localized defect of the aorta resembling human aortic coarctation (Weinstein et al., 1995; Zhong et al., 2000), with Hey2 having a key role in specifying arterial versus venous cell fates (Hermkens et al., 2015; Zhong et al., 2001; Zhong et al., 2000). Of note, Hey2 has been suggested to regulate growth of the heart via restraining cardiomyocyte proliferation (Jia et al., 2007).

Based on computational approaches, Hey2 has recently been predicted to be a key regulator of human cardiac development (Gerrard et al., 2016). Given the evidence linking Hey2 function with CHD-associated defects in various model systems, and the association between CHD and SHF progenitors, we hypothesized that hey2 may have a role in regulating the late-differentiating SHF progenitor pool. Using the zebrafish we were able to discover a distinct domain of hey 2 expression localized to regions adjacent to the myocardium, suggestive of a function for Hey2 in the SHF. Analysis of a novel null hey2 mutant allele revealed increased myocardial cell number, with an apparent increase in the size of the SHF progenitor pool at multiple stages of cardiac development. Temporal analysis demonstrated that progenitor cells underwent increased proliferation prior to, but not following, addition to the heart in the absence of Hey 2 function. This led to both more robust and extended late addition of cardiac progenitors to the heart, with hey 2 acting in a cell-autonomous manner in this context.

94 Taken together, these results suggest that hey2 acts as a key brake on the proliferative capacity and deployment of SHF progenitors to the vertebrate heart. 
EXPERIMENTAL PROCEDURES

98

\section{Zebrafish husbandry and transgenic lines}

100 Adult zebrafish were maintained as per Canadian Council on Animal Care (CCAC)

101 and The Hospital for Sick Children Animal Services (LAS) guidelines. Zebrafish

102 embryos were grown at $28.5^{\circ} \mathrm{C}$ in embryo medium as previously described

103 (Westerfield, 1993). The following transgenic lines were used: $\operatorname{Tg}\left(\right.$ myl7:EGFP) ${ }^{\text {twu34 }}$,

104 (Huang et al., 2003), $\operatorname{Tg}\left(n k x 2.5: Z s Y_{\text {Yellow }}\right)^{f b 7}$ (Zhou et al., 2011),

$105 \operatorname{Tg}(\text { myl7:nlsKikGR })^{\text {hsc6 }}$ (Lazic and Scott, 2011), Tg(myl7:nlsDsRedExpress) $)^{\text {hsc } 4}$ (Lou et

106 al., 2011), Tg(gatal:DsRed) ${ }^{s d 2}$ (Traver et al., 2003) and Tg(myl7:mCherry-RAS) ${ }^{s d 21}$

107 (Yoruk et al., 2012). The hey $2^{\text {hsc } 25}$ mutant line was generated using CRISPR/Cas9

108 genome editing technology as previously described (Jao et al, 2013). Primers

109 TAGGCCAGAAAGAAGCGGAGAG and AAACCTCTCCGCTTCTTTCTGG were

110 annealed together and ligated into the pT7-gRNA vector digested with BsmBI to

111 create sgRNA for hey2. An 8bp deletion allele (starting at nucleotide 151 within

112 exon2) resulting in a premature stop codon at amino acid 53 was isolated (Fig. 3A).

113 An additional allele harboring a 5bp deletion (starting at nucleotide 152 with a

114 premature stop codon at amino acid 55) was isolated showing an equivalent

115 phenotype (data not shown). The hey2 enhancer transgenic epiCon21:EGFP ${ }^{h s c 28}$,

116 containing an epigenetically-conserved open chromatin region (epiCon21), was

117 identified from comparative epigenetic analysis (XY, MDW and ICS, manuscript in

118 preparation). The $626 \mathrm{bp}$ enhancer sequence, located $24 \mathrm{~kb}$ upstream of the hey2 locus

119 (supplemental Fig. 1A), was amplified from zebrafish genomic DNA (FP 5' -

120 CAAATCCCCTGACCTCTGCTTTGAG - 3’; RP 5' -

121 GACACACAGTGACATGTCCTATTGCG - 3') and cloned into the enhancer

122 detection vector E1b-Tol2-GFP-gateway (Addgene 37846 (Li et al., 2010). To

123 generate $T g$ (epiCon21:EGFP), 25ng of E1b-Tol2-GFP-gateway plasmid carrying the

124 epiCon21 enhancer was injected into wild type embryos at the one-cell stage with

125 150ng Tol2 mRNA. Four independent germline carriers were identified, which

126 demonstrated indistinguishable patterns of GFP expression. The epiCon21:EGFP $P^{\text {ss } 28}$

127 was maintained and used for all experiments. Generation of $T g\left(h e y 2 V 5^{h s c 27}\right)$ was

128 performed as previously described (Burg et al., 2016). nCas9n mRNA was injected at 
129 a concentration of $150 \mathrm{pg}$ together with $30 \mathrm{pg}$ of hey $2 \mathrm{gRNA}$ into the yolks of 1-cell

130 embryos. V5 tagging oligo (5' -

131 AGTCATGGCCAGAAAGAAGCGGCAAGCCTATCCCAAACCCTCTGCTGGGC

132 CTGGACTCCACAGGAGAGGGGTAATTCATATT - 3') was diluted to $25 \mu \mathrm{g} / \mu \mathrm{l}$

133 and $1 \mathrm{nl}$ was injected into the yolks immediately after the RNA injections.

\section{Morpholinos}

136 Morpholino oligos were purchased from Genetools (Oregon, USA). A morpholino 137 targeting the translation start site of hey2 (ATG hey2: 5' -

138 TGCTGTCCTCACAGGGCCGCTTCAT - 3') was used throughout this study. The

139 hey2 morpholino (5' - CGCGCAGGTACAGACACCAAAAACT - 3') previously

140 described (Jia et al., 2007) was used to test specificity, as both morpholinos share no

141 sequence overlap. Injection of 1ng of ATG hey2 morpholino at the one-cell stage

142 yielded a consistent heart phenotype.

\section{Standard RNA in situ hybridization}

145 Standard RNA in situ hybridization was performed as previously described (Thisse

146 and Thisse, 2008). The complete coding sequence of hey2 (ZDB-GENE-000526-1)

147 was PCR amplified (sense 5'-ATGAAGCGGCCCTGTGAGGACAGC, antisense 5'-

148 TTAAAACGCTCCCACTTCAGTTCC) and used as a riboprobe template. GFP

149 riboprobe sequence was cloned into pGEM-Teasy and transcribed per standard

150 techniques. Previously described riboprobes were additionally used: hand2 (ZDB-

151 GENE-000511-1), myl7 (ZDB-GENE-991019-3), nkx2.5 (ZDB-GENE-980526-321),

152 mef2cb (ZDB-GENE-040901-7), amhc (ZDB-GENE-031112-1), vmhc (ZDB-GENE-

153 991123-5), tbxl (ZDB-GENE-030805-5), ltbp3 (ZDBGENE-060526-130), bmp4

154 (ZDB-GENE-980528-2059) and $t b \times 2 b$ (ZDB-GENE-990726-27) (Chen and Fishman,

155 1996; Lazic and Scott, 2011; Yelon et al., 1999). DIG and Fluorescein-labeled probes

156 were made using a RNA Labeling Kit (Roche).

\section{Quantitative RT-PCR}

159 Quantitative real-time PCR was performed using the Roche LightCycler 480 with

160 Platinum SYBR green master mix used as per manufactures instructions

161 (ThermoFisher Scientific 11733038). Primers used are as follows: hey2 forward 5' 
GTGGCTCACCTACAACGACA 3', reverse 5' CCAACTTGGCAGATCCCTGT 3'; $m e f 2 c b$ forward 5' CAGCCCAGAGTCAAAGGACA 3', reverse 5', AGGGCACAGCACATATCCTC 3' and $n k x 2.5$ forward 5'

166 Previously described primers were used for quantification of $\beta$-actin (Tang et al., 167 2007); ltbp3 (Zhou et al. 2011); tbxl (Zhang et al., 2006) and amhc (Jia, et al 2007)

168 transcript levels.

\section{Small molecule treatments}

171 The FGF receptor inhibitor SU5402 (Tocris 3300) was used at a concentration of $17210 \mu \mathrm{M}$ from 16.5 to 20 hours post-fertilization (hpf) or from 19 to $24 \mathrm{hpf}$. BMP and 173 Notch signaling inhibitors dorsomorphin (Tocris 3093) and DAPT (Tocris 2634/10), 174 respectively, were used at a concentration of $10 \mu \mathrm{M}$ and $50 \mu \mathrm{M}$ between 16.5 and 20

175 hpf. Retinoic Acid (Sigma R2625) was added at a concentration of $0.1 \mu \mathrm{M}$ to 176 dechorionated embryos at $5.3 \mathrm{hpf}$ ( $50 \%$ epiboly) for 1 hour. All compounds were 177 diluted into $1 \%$ DMSO in embryo medium. Vehicle controls were treated with $1 \%$ 178 DMSO. Incubations were performed at $28^{\circ} \mathrm{C}$.

\section{Imaging}

181 Bright-field images were taken using a Zeiss AXIO Zoom V16. RNA in situ

182 hybridization images were captured using a Leica M205FA microscope with the LAS

183 V6 software package. Immunofluorescence (IF) confocal images were taken with a

184 Nikon A1R laser scanning confocal microscope.

\section{Immunofluorescence, DAF-2DA staining and cell counting}

187 Whole-mount IF was carried out as previously described (Alexander et al., 1998).

188 Primary antibodies used were: $\alpha$-MYH6 supernatant 1:10 (DSHB, S46); $\alpha$-MHC 189 supernatant 1:10 (DSHB, MF20); $\alpha-M E F-2$ (C21) 1:250 (Santa Cruz sc-313); $\alpha$ 190 RCFP 1:400 (Clontech 632475); $\alpha$-Neurolin (cd-166) supernatant 1:10 (DSHB, ZN191 8); $\alpha$-DsRed 1:200 (Clontech 632496); $\alpha$-V5 1:500 (ThermoFisher Scientific R960-

192 25) and $\alpha$-GFP 1:1000 (Torrey Pines Biolabs). Smooth muscle of the bulbus 193 arteriosus was visualized using the NO indicator DAF- 2DA (Sigma D2813) as 194 previously described (Grimes et al., 2006). Cardiomyocyte nuclei of 
myl7:nlsDsRedExpress transgenic embryos were counted following immunostaining.

Embryonic hearts were dissected and flat-mounted prior to confocal imaging.

\section{Photoconversion and cell addition analysis}

199 Photoconversion on myl7:nlsKikGR embryos was carried out as previously described

200 (Lazic and Scott, 2011) using the UV channel on a Zeiss Axio zoom V16 microscope.

201 Images were captured using a Nikon A1R laser scanning confocal. For mounting,

202 embryos were fixed in 4\% PFA for 20 minutes and washed three times in PBS.

203 Embryos were agitated in 5\% saponin/PBS $0.5 \% \mathrm{Tx}-100$ followed by dehydration to

$20475 \%$ glycerol/PBS and left overnight at $4^{\circ} \mathrm{C}$. Hearts were dissected and flat mounted

205 prior to imaging.

206

\section{EdU incorporation}

208 EdU incorporation assays were performed as previously described (Zeng and Yelon,

209 2014). Embryos were incubated in 10mM EdU at 16, 22 and $24 \mathrm{hpf}$ on ice for 30

210 minutes. A Click-iT imaging kit (Invitrogen) was used to visualize EdU

211 incorporation. $\operatorname{Tg}(n k x 2.5: Z s Y e l l o w)$ expression was detected using $\alpha$-RCFP (Clontech

212 632475) with Alexa Fluor 488-conjugated goat anti-rabbit secondary. A proliferation

213 index was calculated based on cells positive for both EdU (red) and ZsYellow (green)

214 staining.

\section{Statistical analysis}

217 Excel software was used to perform student t-tests with two-tail distribution. Graphs

218 display mean \pm s.e.m unless otherwise stated. Box plot graphs were prepared using

219 BoxPlotR web-tool (http://boxplot.tyerslab.com).

\section{RESULTS}

\section{hey2 expression marks a subset of cardiac progenitor cells}

225 To further examine the role of Hey2 in cardiac development, we first carried out a

226 detailed analysis of hey 2 expression during key stages of cardiogenesis, spanning

227 early cardiac specification to the formation of the linear heart tube, using whole- 
mount RNA in situ hybridization. Interestingly, hey2 transcripts were found to localize anteromedial to those of $n k x 2.5$ and $m e f 2 c b$ in the anterior lateral plate mesoderm (ALPM) at 16.5 hours post-fertilization (hpf, Fig. 1A and D, respectively). At $20 \mathrm{hpf}$, when the primitive heart is organized into a cone of differentiating cardiac cells (Yelon et al., 1999), hey2 expression was again evident anteromedial to that of $m y l 7$ and mef2cb (Fig. 1B and E). We further observed a domain of hey2 expression

234 lateral to the heart cone, in the region of the pharyngeal mesoderm (Fig. 1B and E, white arrowheads). Following formation of the linear heart tube at $24 \mathrm{hpf}$, hey2 transcripts were detectable both within and extending from the distal portion of the ventricle, a region occupied by mef $2 c b$-positive cells of the presumptive SHF (Fig. 1C and F; (Lazic and Scott, 2011). These results, as summarized in Figure 1G, suggest that hey 2 is an early marker of the late-differentiating progenitor population, as it is expressed in a manner consistent with regions shown to contain SHF progenitors (Guner-Ataman et al., 2013; Hami et al., 2011).

243 To further dissect the expression of hey 2 with respect to the late-differentiating 244 progenitor population, we pursued both isolation of key hey2 regulatory regions and 245 tagging of the endogenous hey 2 coding sequence. Epigenetic analysis of early zebrafish cardiogenesis identified an enhancer (epiCon21) located 24kb upstream of hey2 that shares an open chromatin signature between zebrafish, mouse and humans (supplemental Fig. 1A; XY, MDW and ICS, manuscript in preparation). Stable $\mathrm{Tg}($ epiCon 21:EGFP) transgenic animals were made in which this enhancer drove GFP expression. Following RNA in situ, analysis of Tg(epiCon21:EGFP) and myl7 expression at 16.5, 20 and $24 \mathrm{hpf}$ showed that the transgenic faithfully recapitulated the endogenous hey2 gene expression pattern (Fig. 1 compare H-J with A-C). Higher resolution analysis revealed $\mathrm{Tg}$ (epiCon21:EGFP $)^{\text {hsc } 28}$ expression at $20 \mathrm{hpf}$ restricted to

254 the anteromedial region of the $\operatorname{Tg}(n k x 2.5 \mathrm{ZsYellow})$-positive heart cone, as was observed by RNA in situ hybridization (Fig. 1 B and K-K' '). The higher resolution afforded by fluorescent immunohistochemistry further indicated $\operatorname{Tg}$ (epiCon21:EGFP) co-expression with $\operatorname{Tg}(n k x 2.5 \mathrm{Zs}$ Yellow) in the pharyngeal mesoderm (Fig. 1K', asterisk; (Paffett-Lugassy et al., 2013). A further domain of Tg(epiCon21:EGFP) expression was also observed immediately anterior to the heart cone, with these cells having no detectable $\operatorname{Tg}(n k x 2.5 Z s Y$ Yllow) expression (Fig. K' ; arrowhead), matching 
261 the position of recently described isl2b-positive SHF cells (Witzel et al., 2017). By 24

$262 \mathrm{hpf}, \operatorname{Tg}(\text { epiCon 21:EGFP })^{\text {hsc28 }}$ shows clear expression within the posterior ventricular

263 region, with the most posterior domain being negative for myl7 expression (Fig. 1L-

264 L"'; arrowheads indicate ventricular portion and arrows indicate atrial portion of the

265 heart tube). Analysis of the expression of $\operatorname{Tg}(\text { epiCon } 21: E G F P)^{h s c 28}$ against that of

$266 \mathrm{Tg}$ (myl7:mCherry) until $94 \mathrm{hpf}$ demonstrated specificity to the ventricular

267 myocardium and OFT, with an absence of detectable expression in the atrium and

268 atrio-ventricular canal (supplemental Fig. 1B-F).

270 To conclusively follow endogenous hey2 expression, we further used CRISPR/Cas9

271 genome editing to place an internal V5 epitope tag into the hey2 locus (supplemental

272 Fig. 1G; Burg et al, 2016). Tg(hey2-V5 $)^{h s c 27}$ embryos were viable, demonstrating that

273 this allele was functional. Antibody staining versus V5 at 30 hpf showed V5-Hey2

274 localization to the posterior ventricular region of the heart tube (Fig. 1M; arrowhead

275 ventricle; arrow atrium), with a portion of CMs expressing both myl7:mCherry and

276 V5 (Fig. 1M; asterisk). This expression data replicates both endogenous expression of

277 hey2 (Fig. 1C) as well as enhancer expression of hey2 (Fig. 1J and L'). Taken

278 together, expression analysis revealed subsets of hey2-positive cells between 15 and

$27920 \mathrm{hpf}$ which 1 ) only express hey2; 2) co-express both hey 2 and $n k x 2.5$ in the cardiac

280 cone; and 3) co-express hey2 and $n k x 2.5$ in presumptive cardiac progenitors within

281 the pharyngeal mesoderm.

Opposing effects of FGF and RA signaling on hey2 expression suggest a link to SHF progenitors.

285 To further explore the link between Hey2 and the late-differentiating progenitor 286 population, we reasoned that hey 2 expression should be affected by modulation of 287 signaling pathways that have been implicated in regulating SHF progenitor 288 development. Both FGF and retinoic acid (RA) signaling have been shown to play 289 opposing roles during cardiac specification, diversifying the heart fields within the 290 ALPM (Ryckebusch et al., 2008; Sirbu et al., 2008; Waxman et al., 2008).

291 Subsequent to this, FGF signaling is required within the late-differentiating SHF 292 progenitors for proper OFT development and maintenance of progenitor proliferation and survival (Ilagan et al., 2006; Park et al., 2008; Zeng and Yelon, 2014). Previous 
294 reports identified hey 2 as a target of both FGF and RA signaling, with RA acting via

295 FGF (Feng et al., 2010; Sorrell and Waxman, 2011).

297 By modulating FGF signaling between $16.5 \mathrm{hpf}$ and $20 \mathrm{hpf}$ using a well characterized

298 inhibitor, SU5402, we noted the expected reduction in ventricular cardiomyocyte

299 differentiation but normal atrial differentiation, as shown by $v m h c$ and $a m h c$

300 expression (supplemental Fig. 2A and B). SU5402 treatment between 16.5 hpf and 20

301 hpf further resulted in reduced cardiac hey2 expression within the heart cone (Fig. 2A

302 and B), with a coincident loss of detectable expression of the SHF progenitor marker

$303 m e f 2 c b$ (supplemental Fig. 2C and D, (Lazic and Scott, 2011). Later addition of

304 SU5402, between 19 and 24 hpf, resulted in a loss of cardiac hey2 expression, yet no

305 overt change to cardiac myl7 or neural hey2 expression (Fig. 2C and D; arrowhead

306 cardiac, asterisk neural). While this result showed coincident loss of SHF-associated

307 progenitors and hey2 expression, we could not preclude that the absence of hey2

308 simply reflected its association with a ventricular fate.

310 To further examine the potential relationship between hey2 expression and SHF

311 progenitors, we next modulated RA activity. The effect of RA on SHF development

312 was studied via use of a photoconversion assay in myl7:nlsKikGR embryos via

313 quantifying later (SHF-derived) addition of cardiomyocytes to the heart tube after 24

314 hpf (Lazic and Scott, 2011). Addition of RA at 4 hpf (50\% epiboly) inhibited

315 myocardial accretion between 24 and $48 \mathrm{hpf}$ as shown by a reduction in green-only

316 cardiomyocytes as well as a significant loss of ventricular CM number (supplemental

317 Fig. 2E-H). This highlights the inhibitory effect of RA on the late-differentiating

318 progenitor population. In keeping with this result, hey2 expression was undetectable

319 under the same conditions of exogenous RA treatment at both 16.5 and $28 \mathrm{hpf}$ (Fig.

320 2E-H). Loss of hey2 expression following RA treatment was accompanied by a

321 reduction in expression of tbxl and hand2 (Fig. 2I-L) as well as a loss of mef $2 c b$

322 expression (supplementary Fig. 2I and J), all of which are associated with SHF

323 development. In contrast, no appreciable effects on $n k x 2.5$ or myl7 expression was

324 observed at either 16.5 hpf (Fig. 2M-P) or 28 hpf (supplemental Fig. 2K and L),

325 suggesting comparatively normal FHF progenitor development. In contrast to FGF

326 and RA signaling, inhibition of BMP and Notch pathways had no detectable effect on 
hey2 expression by $20 \mathrm{hpf}$ (supplemental Fig. 2M and N). While RA treatment affected both ventricular and SHF cell fate at $28 \mathrm{hpf}$, the early effect on expression of hey 2 and other SHF markers suggested a link between hey 2 and the latedifferentiating population.

\section{Cardiovascular defects in the absence of hey2 function}

333 As expression of hey2 was suggestive of a role in early cardiac development, prior to 334 heart tube assembly, we next investigated the effect loss of Hey2 function. Previous reports demonstrated the consequences of knocking down hey2 gene function using either morpholinos (MOs) or with existing hey2 mutants: an ENU mutagenesis allele $\left(\right.$ grl $^{m l 45}$ (Weinstein et al., 1995; Zhong et al., 2000) and in mosaic TALEN-injected embryos (Hermkens et al., 2015). However, overexpression experiments had suggested that the $\mathrm{grl}^{\mathrm{m} 145}$ allele, which encodes a Hey2 protein with a 44aa C-terminal extension, retains some function (Jia et al., 2007; Zhong et al., 2000). With both this and recent controversy regarding use of antisense morpholinos to assess gene function in zebrafish (Kok et al., 2015) in mind, we generated a novel predicted null mutation in hey2 using CRISPR/Cas9-mediated genome editing. By targeting the hey2 transcript within exon2, we generated a mutant with an 8bp deletion producing a premature stop codon at amino acid 53, effectively deleting the bHLH domain (Fig. $3 \mathrm{~A})$. Both hey $2^{h s c 25}$ mutant and morphant phenotypes become apparent by $48 \mathrm{hpf}$ with mutant embryos displaying a non-looped heart with a reduction in heart rate when compared to controls (supplemental Fig. 3A-D). At 72 hpf, hey2 mutant embryos exhibited pericardial edemas accompanied by an enlarged atrium, evident by a significant increase in $a m h c$ gene expression as well as a truncated ventricle (Fig. 3BE, supplemental Fig. 3E-G). As in grl $^{m 145}$ mutants (Zhong et al., 2001), hey2 $2^{\text {hsc25 }}$ mutants demonstrated a blockage at the aortic bifurcation preventing blood flow to

353 the trunk, evident by the slow movement of gatal + cells in the trunk vasculature (Fig.

$3543 \mathrm{~F}$ and $\mathrm{G}$, arrowhead). As hey2 loss-of-function demonstrated a late morphological 355 phenotypic onset, early developmental stages were analyzed using hey 2 morpholino 356 to facilitate these studies.

\section{Loss of Hey2 affects cardiac function and maturation}


359 Given the irregular morphology of hey2 mutant hearts, we next investigated

360 cardiomyocyte-intrinsic defects. The function of the heart is highly dependent on

361 cardiomyocyte morphology, specifically cell shape (Auman et al., 2007).

362 Cardiomyocytes initially have a uniform cuboidal characteristic that is altered as

363 ventricular chamber formation proceeds by both blood flow and cardiac contractility

364 (Manasek, 1981; Manasek et al., 1972; Taber, 2006). To examine potential defects in

365 cardiac maturation, the hey $2^{h s c 25}$,allele was crossed into a $T g$ (myl7:nlsDsRedExpress)

366 background. IF staining using antibodies against DsRed and zn-8 was used to

367 visualize cardiomyocyte cell nuclei and cell membranes, respectively. As compared to

368 sibling controls, in hey $2^{h s c 25}$ mutants cardiomyocytes failed to initiate cellular

369 elongation near the atrio-ventricular canal (AVC) (Fig. 3H and I, arrowheads).

370 Instead, cell shape remained uniform throughout the ventricle. This was quantified

371 through axis ratio measurements, which showed a significant difference in cell shape

372 at the AV boundary in hey $2^{h s c 25}$ mutants compared to control embryos (Fig. 3J). To

373 further characterize the defects observed within the AVC of hey $2^{\text {hsc } 25}$ mutants, we

374 performed RNA in situ hybridization. Upon AVC differentiation, $t b \times 2 b$ and $b m p 4$

375 expression become restricted to the AVC, with no transcript detected in the chamber

376 myocardium (Rutenberg et al., 2006). We found that in hey $2^{h s c 25}$ mutants bmp4 and

$377 t b \times 2 b$ transcripts remained distributed in the chambers, with no characteristic

378 restriction to the $\mathrm{AVC}$, a phenomenon also reported in mouse embryos misexpressing

379 hey2 (supplemental Fig. 3H-K; (Kokubo et al., 2007). These results suggest a function

380 for Hey2 in proper AVC development and cardiac maturation.

\section{Expanded cardiomyocyte number in hey2 mutants}

383 Based on the expression of hey2 prior to $24 \mathrm{hpf}$, we next analyzed the structure of the

384 heart tube between wild type and hey2 MO injected embryos. At 26 hpf, hey2

385 morphants had an irregularly shaped heart that had failed to elongate (Fig. 4A-D),

386 with an expansion in expression of terminal differentiation markers myl7 and tnnt 2 at

387 the poles of the heart (Fig. 4B and D, arrowheads). Given this observation, we

388 counted CM number in wild type and hey2 morphant Tg(myl7nls:DsRedExpress)

389 embryos. Whereas control embryos contained 136.8 \pm 6 (mean \pm s.e.m, $n=5$ ) CMs at 24

390 hpf, hey2 morphant embryos contained a significantly greater number $(170 \pm 3.6$,

391 mean \pm s.e.m, $n=5$; Fig. 4E-G). As the heart develops, the number of CMs increases 
significantly between 24 and 48 hpf (de Pater et al, 2009). We therefore next determined the effect of hey2 loss on $48 \mathrm{hpf} \mathrm{CM}$ number. As observed at $24 \mathrm{hpf}$, there was a significant increase in myl7:nlsDsRedExpress-positive CM nuclei in the absence of hey 2 as compared to controls $\left(238.7 \pm 2.4\right.$ in hey $2^{\text {hsc } 25}$ mutants; $183 \pm 1.5$ in controls, mean \pm s.e.m, $n=6$; Fig. 4F-H). These results highlight that in the absence of hey2, an elevated addition of CMs to the heart is detectable as early as $24 \mathrm{hpf}$.

\section{Increased proliferation of cardiac progenitors contributes to increased heart size} in the absence of hey2 function

Given the increased CM number observed in hey2 deficient embryos from as early as $24 \mathrm{hpf}$, we explored the hypothesis that this may be due to accelerated addition of cardiac progenitors to the heart. Using whole-mount in situ hybridization and quantitative RT-PCR, we observed a significant increase in the expression of $n k x 2.5$, mef2cb, ltbp3 and hey2 in hey 2 morphants compared to WT controls within the $24 \mathrm{hpf}$ linear heart tube (Fig. 5A-J). At 48 hpf, we observed through IF staining a higher number of Mef2-positive cells at the arterial pole of the two-chambered heart (supplemental Fig. 4C-F). As we noted that the effect of hey2 loss-of-function on CM number is evident as early as $24 \mathrm{hpf}$, we next analyzed embryos at $16.5 \mathrm{hpf}$ for gene expression changes within the ALPM. Interestingly, we observed increased expression of $n k x 2.5$ (Fig. 5K, N and R) as well as broader expansion of $m e f 2 c b$ (Fig. 5L, O and Q) and hey2 (Fig. 5M, P and S) expression domains. This expansion in early cardiac mesoderm gene expression was evident from as early as $12 \mathrm{hpf}$ (supplemental Fig. 4A and B), implying that in the absence of hey2 there is an expansion in the cardiac progenitor population. These results suggest that hey 2 plays an early, previously unappreciated role in restricting the size of the early cardiac progenitor population, in particular that which expresses markers of the SHF (mef2cb, tbxl and ltbp3).

The upregulation of SHF-associated genes observed following hey2 loss-of-function led us to examine if there was increased proliferation of cardiac progenitors that were later added to the developing heart. Although CMs have the potential to proliferate, minimal proliferative activity has been observed within the myocardium between 24 and 48 hpf (de Pater et al., 2009). We therefore first assessed proliferation of cardiac 
progenitors prior to $24 \mathrm{hpf}$. These progenitors are believed to reside within pharyngeal mesoderm, in an area demarcated by $t b x l$ expression, which has been shown to regulate SHF development (Buckingham et al., 2005; Chapman et al., 1996; Hami et al., 2011; Nevis et al., 2013). Tbx1 has been shown to promote proliferation of SHF progenitors and maintain the cardiac progenitor pool as myocardial accretion occurs

430 (Nevis et al., 2013). As our initial analysis suggested that hey2 (epiCon21:EGFP ${ }^{\text {hsc } 28}$ )

431 is co-expressed with $\mathrm{Tg}(n k x 2.5 \mathrm{Zs}$ Yellow $)$ expressing cells within the pharyngeal 432 mesoderm (Fig. 1K", asterisk), we examined tbxl expression in hey 2 morphant 433 embryos and found an increase in tbxl transcript levels at $16.5 \mathrm{hpf}$ (Fig. 5T, $\mathrm{U}$ and $434 \mathrm{X})$. Analysis at $20 \mathrm{hpf}$ revealed a partial overlap of hey 2 and $t b x \mathrm{l}$ expression domains 435 within the pharyngeal mesoderm, with no $t b x 1$ expression detectable within the cardiac cone, consistent with previous reports (Fig. 5V, arrowheads; (Nevis et al., 2013). Strikingly, in the absence of hey2 function, both hey 2 and $t b x l$ transcripts were significantly up regulated within the pharyngeal mesoderm (Fig. $5 \mathrm{~W}$, W' compare to V). As Tbx1 regulates SHF proliferation, we next performed an EdU incorporation assay to monitor the proliferative activity of SHF progenitors following hey2 knockdown. $\operatorname{Tg}(n k x 2.5 \mathrm{Zs}$ Yellow $)$ embryos were injected with or without hey2 MO and pulsed with EdU at $16.5 \mathrm{hpf}$ to label proliferating cells. A proliferation index was subsequently determined by comparing the number of EdU+ and EdUcardiomyocytes at $35 \mathrm{hpf}$ in control and hey2 morphant hearts. Hey2 morphant embryos displayed a significantly higher proliferative index as compared to controls, indicating that an increase in proliferation prior to heart tube formation contributes to enhanced CM production (Fig. 5Y-Y', ). In contrast, pulsing with EdU at $24 \mathrm{hpf}$ demonstrated no significant impact of hey2 loss on proliferation in CMs between 2435 hpf (supplemental Fig. 4G-I). Altogether, these results suggest an early role for Hey2 in establishing the appropriate number of late-differentiating progenitors that

\section{Myocardial accretion is extended in hey2 loss-of-function embryos}

454 Previous work has demonstrated and quantified the differentiation and addition of 455 SHF progenitors to the heart tube between 24 and 48 hpf (de Pater et al., 2009; Hami 456 et al., 2011; Lazic and Scott, 2011). In order to better understand the consequences of 457 having more SHF progenitors following loss of Hey2 function, we employed 
458

459

460

461

462

463

464

465

466

467

468

469

470

471

472

473

474

475

476

477

478

479

480

481

482

483

484

485

486

487

488

489

myl7:nlsKikGR embryos to photoconvert cardiomyocytes at $24 \mathrm{hpf}$ and monitor subsequent novel myocardial addition to the heart. We observed a substantial increase in the number of differentiated CMs being added to the arterial pole in hey2 morphants $(95 \pm 7.0)$ compared to WT controls $(23.4 \pm 1.6)$, as shown by green only cells at the arterial pole of the 48 hpf heart (Fig. 6A-D, brackets; mean \pm s.e.m, n=5). The normal timing of termination for myocardial addition has been reported to be between 36 and 48 hpf (Jahangiri et al., 2016; Lazic and Scott, 2011). Due to the increase in cell addition between 24 and 48 hpf, we wondered whether myocardial accretion was extended temporally in the absence of Hey2. By photoconverting embryos at $48 \mathrm{hpf}$, with subsequent imaging at $60 \mathrm{hpf}$, we noted SHF-mediated accretion in control embryos was minimal, consistent with previous findings (Fig. 6F and H, 11.9 \pm 0.7 , mean \pm s.e.m, n=7; (de Pater et al., 2009; Jahangiri et al., 2016). In hey 2 deficient embryos a significantly increased cell addition was evident beyond 48 hpf (Fig. 6G and H, 20.3 \pm 1.3 , mean \pm s.e.m, $n=7$ ). This highlights that in the absence of Hey2, the window of myocardial accretion from the late-differentiating progenitor population becomes extended. Following cell addition to the heart, a subpopulation of SHF progenitors has shown to gives rise to the OFT, a structure containing both myocardium, for lengthening the distal cardiac portion, as well as smooth muscle at the myocardial-arterial junction (Choi et al., 2013; Hami et al., 2011; Waldo et al., 2005; Zeng and Yelon, 2014; Zhou et al., 2011). Given that loss of hey2 resulted in increased proliferation and extended the window of myocardial accretion by cardiac progenitors, we wondered what effect this may have on other lineages of the SHF, in particular the OFT smooth muscle. Via incubation of wild type and hey $2^{h s c 25}$ mutant embryos in DAF-2DA, a compound which specifically labels smooth muscle of the OFT (Grimes et al., 2006), we found that hey $2^{h s c 25}$ mutants had a significantly longer OFT than that of sibling controls (Fig. 6I-K). Together this demonstrates that in the absence of Hey2, late-differentiating progenitors are subjected to an extended window of accretion following an increase in proliferative activity, which ultimately leads to an expansion in SHF-derived structures.

\section{hey2 acts cell autonomously to regulate SHF addition to the heart at the expense} of the FHF population 
490 We next employed a transplantation approach to examine the cell autonomy of Hey2

491 activity in cardiac progenitors. To accomplish this, $T g(m y l 7: n l s K i k G R)$ donor

492 embryos, either WT or injected with hey2 MO, were used. Donor cells at $4 \mathrm{hpf}(50 \%$

493 epiboly) were transplanted to the margin of WT host embryos (Fig. 7A), an approach

494 that has been shown to result in cardiac contribution of donor cells (Scott et al., 2007;

495 Stainier et al., 1993). Transplant embryos were photoconverted at either 24 or $48 \mathrm{hpf}$

496 and imaged at either 48 or $60 \mathrm{hpf}$ (Fig. 7B and F, respectively). From our results we

497 observed that Hey2 morphant donor $\operatorname{Tg}($ myl7:nlsKikGR) cells displayed a significant

498 increase in SHF contribution (shown by the ratio of green:yellow cardiomyocytes per

499 embryo), as compared to early myocardial addition, between 24 to $48 \mathrm{hpf}$ (Fig. 7C-E).

500 The same result was observed between 48 to $60 \mathrm{hpf}$, with Hey2 deficient

501 myl7:nlsKikGR donors contributing significantly more green only cardiomyocytes

502 than controls (Fig. 7F-I). While the ratio in numbers of late versus early differentiated

503 cardiomyocytes per heart from hey2 donor cells was consistently increased at both 24-

50448 and 48-60 hpf, an analysis of cell numbers for each category revealed a bias in

505 progenitor populations. From $24-48 \mathrm{hpf}$, the increased late versus early CM addition

506 ratio observed in hey 2 morphants was due to a decreased propensity for donor cells to

507 contribute early to the heart, evident by a significant decrease in total cell number of

508 yellow CMs in controls compared to morphant embryos (Fig. 7J). However, when

509 comparing total number of late differentiating CMs, no statistical significance was

510 observed (Fig. 7J). In contrast, the higher late versus early addition ratio observed at

$51148 \mathrm{hpf}$ was due to a significantly higher amount of late (post $48 \mathrm{hpf}$ ) CM addition,

512 with a relatively equivalent amount of early (pre $48 \mathrm{hpf}$ ) CM addition as noted by no

513 significant change in early CM number between control and hey2 morphant embryos

514 (Fig. 7K). These results demonstrate a cell-autonomous function for Hey2, in

515 presumptive cardiac progenitors, that delays their addition to the heart as

516 cardiomyocytes.

\section{DISCUSSION}

519 Our work demonstrates a novel role for the bHLH factor Hey2 in regulating the size

520 of the cardiac progenitor pool and the timing of the contribution of late-differentiating

521 cardiac progenitors to the zebrafish heart. As shown by fate mapping (Camp et al.,

522 2012; Mjaatvedt et al., 2001) and lineage tracing (Cai et al., 2003; Meilhac et al., 
523 2003) approaches, the vertebrate heart is made via the temporally distinct addition of

524 at least two populations of cardiac progenitors. A recent study using live imaging of

525 cell lineage tracing and differentiation status suggests that in mouse a discrete

526 temporal lag can be observed between the first and second waves of differentiation

527 that form the heart (Ivanovitch et al., 2017). Whether the cardiac progenitor pools that

528 are added early and late to the heart, termed the FHF and SHF, represent molecularly

529 distinct populations remains an open question. While FHF- and SHF-restricted cells

530 can be identified at early gastrulation stages in mouse by clonal analysis (Devine et

531 al., 2014; Lescroart et al., 2010), this may reflect the result of distinct migratory paths

532 and signaling milieus experienced by cells during gastrulation.

534 How the relative size of cardiac progenitor pool(s), the timing of their differentiation

535 and the extent to which they are added to various components of the heart all remain

536 largely unknown. Our work has uncovered a cell autonomous function for Hey2 to

537 restrain proliferation of cardiac progenitors prior to their addition to the heart. Our

538 expression analyses suggest that hey 2 co-localizes with previously identified SHF-

539 associated genes including isl2 $b$ and mef2 $c b$ (Figure 1; (Lazic and Scott, 2011; Witzel

540 et al., 2017) and is in agreement with fate mapping work that has shown SHF

541 progenitors to reside in an anteromedial position within the ALPM (Hami et al.,

542 2011). Importantly, we and others have shown that hey2 expression is regulated not

543 by a canonical Notch signaling pathway, but by RA/FGF signaling (Sorrell and

544 Waxman, 2011). This is consistent with the Notch-independent, FGF-mediated

545 expression of Hey2 in other developmental contexts (Doetzlhofer et al., 2009).

546 Previous reports have highlighted that the cardiac malformations found in animals

547 lacking Hey2 function resemble common human congenital heart defects including

548 ventricular septal defects, tetralogy of fallot and tricuspid atresia (Donovan et al.,

549 2002). Coupled with the expression of hey2 during cardiac development, the effects

550 of hey2 loss on late myocardial addition to the zebrafish heart suggest a mechanism

551 where Hey2 acts specifically in SHF progenitors. While we did not observe

552 appreciable effects on FHF-associated markers in our study, this data is difficult to

553 interpret, as bona fide FHF- and SHF-specific markers that distinguish these

554 populations are poorly characterized. Our transplant assays strongly suggest that

555 cardiac contribution is at the very least delayed with the loss of hey2. The observed 
phenotypes may reflect: 1) a shift in the balance between FHF and SHF progenitor proliferation, favouring the SHF pool; 2) alteration in the timing of cardiac progenitor differentiation; 3) changes in the number of progenitors allocated to the FHF and SHF pools; or 4) a FHF/SHF-agnostic role for Hey2 in cardiac progenitor proliferation and differentiation. This is a critical question that will require further study, but that may provide insight into the diversity of early cardiac progenitors.

563 It is important to note that the function of hey 2 in zebrafish cardiogenesis has been

564 previously addressed in an elegant study (Jia et al., 2007). However, while the overall "large heart" phenotype observed is shared between our hey $2^{\text {hsc25 }}$ and the grl $^{\text {ml45 }}$ mutants, our data suggests a role for hey 2 prior to $24 \mathrm{hpf}$, in cardiac progenitors, that subsequently impacts cardiac development. In contrast, Jia and colleagues reported that $g r l$ had minimal affect during this time, with $g r l^{m l 45}$ mutants having comparable cardiomyocyte numbers to controls at $24 \mathrm{hpf}$. This discrepancy may reflect the nature of the hey 2 alleles used, with the hey $2^{h s c 25}$ allele being, we believe, a true null. As the

571 SHF and late myocardial addition in zebrafish had not been described at the time of

572 the prior study, this would have also affected the interpretation of the results. This

573 highlights the fact that Hey2 likely acts at multiple steps of heart development.

575 The myocardium of the AVC is important for the development of the AV cushion and

576 AV node, both derivatives of the SHF (Kelly, 2012). In zebrafish, $b m p 4$ and $t b x 2 b$ are expressed in the AV myocardium, and play critical roles in the establishment of AVC identity (Ma et al., 2005; Zhang and Bradley, 1996). Here we demonstrate the importance of Hey2 in AVC development. Although previous work revealed that the $g r l^{m l 45}$ mutant shows an ectopic expansion of bmp4 expression at $48 \mathrm{hpf}$, little change was observed in $t b x 2 b$ transcripts (Rutenberg et al., 2006). However our mutant $h e y 2^{h s c 25}$ allele revealed upregulation in both $b m p 4$ and $t b x 2 b$, thus providing insight into a potential regulatory network by which hey2 expression in ventricular myocardium constrains $b m p 4$ expression to the AVC, which in turn activates $t b x 2 b$ expression (supplemental Fig. 4L). In the absence of functional Hey2, this repression 586 is absent, resulting AVC-specific genes expanding their expression domains into the 587 cardiac chambers (supplemental Fig. 4M). 
589 Our data suggest multiple roles for Hey2 in cardiogenesis, first acting to restrain

590 cardiac progenitor proliferation (and potentially affecting diversification of FHF and

591 SHF progenitors), and later affecting the timing of when SHF progenitors are added

592 to the developing heart. Hey2 may therefore be an intrinsic regulator of the extent and

593 differentiation of the cardiac progenitor pool, possibly acting as a readout of extrinsic

594 (RA and FGF) niche signals. It remains to be determined if this strictly reflects a role

595 for Hey2 in SHF progenitors, or if Hey2 plays a broader role in all cardiac

596 progenitors. To address these questions, lineage tracing approaches will be required,

597 for which the novel hey2 enhancer transgenics we have uncovered will be of great

598 utility. Given the cell autonomous function of Hey2, identifying its transcriptional

599 targets will also be of great interest. Given the role of Hey2 in restraining cardiac

600 progenitor proliferation, its down-regulation by RA, and the known role of epicardial

601 RA signaling in zebrafish heart regeneration (Kikuchi et al., 2011), a potential role for

602 Hey2 in regeneration should also be investigated. Dissecting how Hey2 regulates

603 cardiac development will help address key unanswered questions with respect to the

604 regulatory mechanisms that coordinate the size and differentiation timing of cardiac

605 progenitors to allow for proper heart development to proceed.

606

607 ACKNOWLEDGMENTS

608 We would like to thank Angela Morley and Allen Ng for expert fish care and 609 maintenance. Neil Chi kindly provided the myl7:EGFP and Caroline and Geoffrey

610 Burns the $n k x 2.5 Z$ zYellow transgenic lines. NG and XY were kindly supported by a

611 Labatt Family Heart Centre Philip Witchel post-doctoral fellowship and a Hospital for

612 Sick Children Restracomp studentship, respectively. Research funding was

613 generously provided by the Heart and Stroke Foundation of Canada (to ICS and

614 MDW, Grant-in-Aid G-16-00013798), the Natural Sciences and Engineering

615 Research Council of Canada (to ICS, RGPIN 341545-12) and the Canadian Institutes

616 of Health Research (Operating Grant MOP-123223 (to ICS) and Project Grant PJT -

617153343 (to ICS and MDW)). 
618

619

620

621

622

623

624

625

626

627

628

629

630

631

632

633

634

635

636

637

638

639

640

641

642

643

644

645

646

647

648

649

650

\section{FIGURE LEGENDS}

Figure 1. hey2 resides in the late-differentiating progenitor population

(A-F) Double RNA in situ hybridization analysis of hey2 (blue) against $n k x 2.5$, myl7 and mef $2 c b$ (orange) in wild type embryos from $16.5 \mathrm{hpf}$ to $24 \mathrm{hpf}$. (G) Schematic representation of FHF and SHF progenitor localization and movement patterns from 16.5 to $24 \mathrm{hpf}$. (H-J) Double RNA in situ hybridization analysis of $\operatorname{Tg}\left(\right.$ epiCon 21:EGFP) ${ }^{h s c 28}$ (blue) and myl7 (orange) at $15 \mathrm{hpf}(\mathrm{H}), 20 \mathrm{hpf}(\mathrm{I})$ and $24 \mathrm{hpf}$ (J). (K-L) Immunofluorescence showing $\operatorname{Tg}(\text { epiCon } 21: E G F P)^{h s c 28}$ enhancer expression against $\operatorname{Tg}(n k x 2.5: Z$ sYellow) at $20 \mathrm{hpf}$ (K-K'”) and $24 \mathrm{hpf}$ (L-L'”). (M) Immunofluorescence of $T g(h e y 2-V 5)^{h s c 27}$ internal epitope tagging with $\operatorname{Tg}(m y l 7: m C h e r r y-R A S)$ at 30 hpf comparing Hey2 expression (green) with Myl7 (red). Scale bars $50 \mu \mathrm{m}$. Asterisk labels pharyngeal mesoderm. Arrows denote the atrium and arrowheads mark the ventricle.

\section{Figure 2. Opposing effects of FGF and RA signaling regulate hey2 expression}

(A-D) Double RNA in situ hybridization analysis of hey2 (blue) and myl7 (orange)

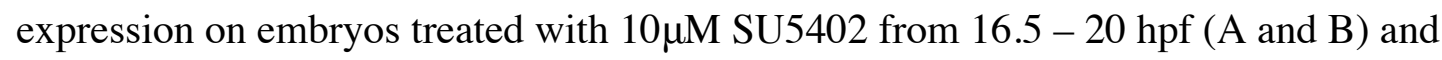
from 19 - 24 hpf (C and D). (E-P) RNA in situ hybridization analysis for hey2 (E-H), tbxl (I and $\mathrm{J})$, hand2 (K and $\mathrm{L})$, myl7 $(\mathrm{M}$ and $\mathrm{N})$ and $n k x 2.5(\mathrm{O}$ and $\mathrm{P})$ following treatment with RA at $0.1 \mu \mathrm{M}$ for 1 hour at $50 \%$ epiboly. Note down regulation in $t b x 1$ and hand 2 but no change in $m y l 7$ and $n k x 2.5$. Asterisks depict neural expression, arrowheads cardiac expression.

Figure 3. Cardiovascular defects are observed in the absence of Hey2 function. (A) Schematic representation of hey2 null-mutant generated through CRISPR/Cas9 mediated genome-editing. Red lettering shows $8 \mathrm{bp}$ deleted sequence. Protein sequence shows production of premature stop codon at the beginning of exon 2. (B,

C) Bright-field images of a sibling control and a hey $2^{\text {hsc } 25}$ mutant embryo at $72 \mathrm{hpf}$. (D,

E) Confocal images of $T g(m y l 7: E G F P)$ hearts in control (D) and hey $2^{\text {hsc } 25}-/-$

embryos at 72 hpf. (F, G) Fluorescent images of $T g$ (gatal:DsRed) showing normal blood flow in controls at $72 \mathrm{hpf}(\mathrm{F})$ and lack of blood flow leading to coagulated blood cells in hey $2^{\text {hsc25 }}$-/- (G, arrowhead). (H-I) Confocal imaging of Tg(myl7:nlsDsRedExpress) embryos co-stained with zn-8 to display cell number 
651 (DsRed) and cell membrane (zn-8) formation in control (H) and hey $2^{\text {hsc } 25}$ mutant

652

653 embryos (I) at $48 \mathrm{hpf}$. (J) Bar graph showing axis ratios of cardiomyocytes taken from the ventricle and the AV boundary between control and hey $2^{h s c 25}$ mutant embryos. $\mathrm{N}=3, \mathrm{n}=10$ per condition. Error bar mean \pm s.e.m; *** $\mathrm{p}<0.001$; n.s, not significant. Scale bar $50 \mu \mathrm{m}(\mathrm{B}-\mathrm{G})$ and $100 \mu \mathrm{m}(\mathrm{H}$ and $\mathrm{I})$.

\section{Figure 4. The absence of Hey2 results in inappropriate cardiomyocyte number} (A-D) RNA in situ hybridization analysis for myl7 (A, B) and tnnt2 (C, D) in control and hey2MO embryos. (E, F) Confocal images of cardiomyocyte nuclei in control and hey2MO embryos using Tg(myl7:nlsDsRedExpress) at 24 hpf. (G) Bar graph showing total cardiomyocyte number at $24 \mathrm{hpf}$ between control and hey2MO embryos $(\mathrm{N}=3$, $\mathrm{n}=5$ per condition). (H, I) Confocal images showing cardiomyocyte nuclei at $48 \mathrm{hpf}$ control (H) and hey $2^{\text {hsc } 25}$ mutant embryos (I). (J) Bar graph showing cardiomyocyte number between atrium and ventricle in control and hey $2^{h s c 25}$ mutant embryos at $48 \mathrm{hpf}$ $(\mathrm{N}=3, \mathrm{n}=6$ per condition). Scale bars $50 \mu \mathrm{m}$. Error bar mean \pm s.e.m; $* * \mathrm{p}<0.01$; *** $\mathrm{p}<0.001$. A, atrium; V, ventricle; OFT, outflow tract.

\section{Figure 5. Hey negatively regulates the expression of SHF-associated genes} (A-F) Riboprobe staining for SHF markers $n k x 2.5$, ltbp3 and $m e f 2 c b$ at $24 \mathrm{hpf}$ in control (A-C) and hey2MO embryos (D-F). (G-J) Quantitative RT-PCR analysis at 24 hpf comparing mef2cb, ltbp3, nkx2.5 and hey2 gene expression in controls to hey2MO embryos. (K-P) RNA in situ hybridization analysis at $16.5 \mathrm{hpf}$ in control and hey2MO embryos for $n k x 2.5, m e f 2 c b$ and combined hey2 (blue) and $n k x 2.5$ (orange). (Q-S) Quantitative RT-PCR analysis for $m e f 2 c b, n k x 2.5$ and hey2 gene expression at 16.5 hpf. (T-W') RNA in situ hybridization analysis of $t b x \mathrm{l}$ at $16.5 \mathrm{hpf}$ (T and U) and coexpression of hey2 (blue) and tbxl (orange) at $20 \mathrm{hpf}(\mathrm{V}$ and $\mathrm{W}, \mathrm{W}$ ') in controls (T, $\mathrm{V})$ and hey2MO (U, W) embryos. (W') Enlarged view of $t b x 1$ and hey2 expression within the pharyngeal mesoderm. (X) Quantitative RT-PCR analysis at $16.5 \mathrm{hpf}$ for tbxl expression. (Y-Y') EdU incorporation (red) in control (Y) and hey2MO (Y') embryos expressing $\operatorname{Tg}(n k x 2.5 Z$ SYellow) (green). EdU positive cardiomyocytes are shown as yellow cells. (Y') Proliferation index between control and Hey2 morphant embryos following EdU pulse at $16.5 \mathrm{hpf}(\mathrm{N}=2, \mathrm{n}=7)$. Error bars, mean \pm s.e.m; *** $\mathrm{p}<0.001$. 
Figure 6. Hey restricts SHF cell addition to the developing heart.

(A) Schematic representation of cardiomyocyte photoconversion assay between 24 and 48 hpf. (B-C) Confocal imaging of control and hey2MO Tg(myl7:nlsKikGR) embryos. Brackets highlight green only SHF-derived cardiomyocytes. (D) Bar graph showing a significant increase in green-only cardiomyocytes at $48 \mathrm{hpf}$ in control compared to Hey2 morphant embryos $(\mathrm{N}=3, \mathrm{n}=7)$. (E) Schematic representation of photoconversion assay between 48 and $60 \mathrm{hpf}$. (F-G). Confocal imaging of control and hey2MO $\operatorname{Tg}(m y l 7: n l s K i K G R)$ embryos showing an increase in SHF-derived green only cardiomyocytes in morphants $(\mathrm{G})$ compared to controls $(\mathrm{F})$. (H) Bar graph showing mean values of green-only cells between 48 and $60 \mathrm{hpf}(\mathrm{N}=3, \mathrm{n}=7)$. (I-K) DAF2-DA labeling of the OFT smooth muscle in control and hey $2^{h s c 25}$ mutant embryos at $72 \mathrm{hpf}$. (K) Bar graph represents mean OFT lengths in control and hey $2^{h s c 25}$ mutant $(\mathrm{N}=2, \mathrm{n}=11$ per condition). Error bars mean \pm s.e. $\mathrm{m} ; * * \mathrm{p}<0.01$; *** $\mathrm{p}<0.001$. Scale bars $50 \mu \mathrm{m}$.

702 (A) Schematic representation of transplantation strategy. (B-D) photoconversion

$703 \mathrm{Tg}(\mathrm{myl} / \mathrm{nlsKiKGR})$ at $24 \mathrm{hpf}$, imaged at $48 \mathrm{hpf}$ in control (C) and hey2MO (D)

704 transplanted embryos. (E) Boxplot analysis demarking the percentage ratio at 24-48

705 hpf between green and yellow transplanted cardiomyocytes in control and hey2MO

706 embryos ( $\mathrm{n}=13$, control and $\mathrm{n}=7$, hey2MO). (F-H) photoconversion at $48 \mathrm{hpf}$, imaged 707 at $60 \mathrm{hpf}$ of $T g(m y l 7: n l s K i K G R)$ control (G) and hey2MO (H) transplants. (I) Boxplot 708 analysis demarking the percentage ratio at 48-60 hpf between green and yellow 709 transplanted cardiomyocytes in control and hey $2 \mathrm{MO}$ embryos $(\mathrm{n}=8$, control and $\mathrm{n}=9$, 710 hey2MO). (J-K) Boxplot analysis displaying total cell number of transplanted

$711 \mathrm{Tg}($ myl7:nlsKikGR) control and hey2MO cardiomyocytes at 24-48 hpf (J; n=12 WT 712 green, $\mathrm{n}=15 \mathrm{WT}$ yellow; $\mathrm{n}=7$ hey2MO green, $\mathrm{n}=7$ hey2MO yellow) and 48-60 hpf (K; $713 \mathrm{n}=8$ WT green, $\mathrm{n}=8 \mathrm{WT}$ yellow; $\mathrm{n}=7$ hey2MO green, $\mathrm{n}=7$ hey2MO yellow). Green 714 cells refer to SHF contribution; yellow cells refer to FHF contribution. Error bars, 715 mean \pm s.e.m; $* * \mathrm{p}<0.01, * * * \mathrm{p}<0.001 ; \mathrm{n} . \mathrm{s}$, no significant difference. Scale bars $50 \mu \mathrm{m}$. 


\section{Supplemental Figure 1, related to Figure 1}

718 (A) Schematic representation of hey2 enhancer (epigenetic conserved region 21)

719 located $24 \mathrm{~kb}$ upstream of hey2 locus used to create $\operatorname{Tg}(\text { epiCon } 21: E G F P)^{\text {hsc } 28}$. (B-F)

720 Confocal microscopy of $T g(\text { epiCon } 21: E G F P)^{h s c 28}$ at $48 \mathrm{hpf}(\mathrm{B}), 72 \mathrm{hpf}(\mathrm{C}, \mathrm{D})$ and 96

721 hpf (E, F) against Tg(myl7:nlsDsRedExpress). (G) Schematic methodology of internal

722 epitope tagging of V5 into hey2 locus with corresponding sequence of tag-specific

723 PCR fragments. Scale bars $50 \mu \mathrm{m}$. OFT, outflow tract; V, ventricle and A, atrium;

724 Asterisk, atrioventricular canal. Arrowheads mark the epiCon21+ OFT region.

\section{Supplemental Figure 2, related to Figure 2}

727 (A-D) double in situ hybridization analysis for amhc (blue), vmhc (orange) and $728 m e f 2 c b$ (blue) at $20 \mathrm{hpf}$ following treatment with $10 \mu \mathrm{M}$ SU5402 from $16.5 \mathrm{hpf}$. (E-F) 729 Confocal imaging at $48 \mathrm{hpf}$ of photoconverted $T g(m y l 7: n l s K i k G R)$ embryos at $24 \mathrm{hpf}$ 730 following treatment with 0.1uM RA at 50\% epiboly. (G-H) Bar graph analysis

731 showing the number of green-only SHF cell addition by $48 \mathrm{hpf}(\mathrm{G})$ and total 732 ventricular cell number $(\mathrm{H}) . \mathrm{N}=2, \mathrm{n}=5$ per condition. Error bars mean $\pm \mathrm{sem}$; *** $733 \mathrm{p}<0.001$. Scale bars $50 \mu \mathrm{m}$.

\section{Supplemental Figure 3, related to Figure 3}

736 (A-C) Bright-field images of $T g(m y l 7: E G F P)$ in control (A), hey $2^{h s c 25}$ mutant (B) and hey2MO (C) embryos at $48 \mathrm{hpf}$. (D) Heart rate analysis represented as beats per minute (bpm) at $48 \mathrm{hpf}$ in control, hey2MO and hey $2^{h s c 25}$ mutant embryos $(\mathrm{N}=3, \mathrm{n}=4)$. (E-F) MF20/S46 immunofluorescence imaging at $72 \mathrm{hpf}$ in control (E) and hey $2^{\text {hsc25 }}$ mutant embryos (F). (G) Quantitative RT-PCR analysis for amhc gene expression in control and hey $2 \mathrm{MO}$ embryos at $48 \mathrm{hpf}$ (gene expression normalized to $\beta$-actin, fold

742 difference relative to control; $\mathrm{N}=3, \mathrm{n}=3)$. $(\mathrm{H}-\mathrm{K})$ Riboprobe staining for $b m p 4(\mathrm{H}$ and

$743 \mathrm{~J})$ and $t b \times 2 b$ (I and $\mathrm{K})$ at $48 \mathrm{hpf}$ in control and hey $2^{h s c 25}$ mutant embryos. (L-M)

744 Schematic representation showing boundary constraints of $b m p 4$ and $t b x 2 b$ genes

745 expression within the ventricular myocardium and AVC in the presence (L) and 746 absence $(\mathrm{M})$ of hey2. ${ }^{* * *} \mathrm{p}<0.001, * * \mathrm{p}<0.01$, error bars mean \pm s.e.m. Scale bars $74750 \mu \mathrm{m}$. 
750 (A-B) Riboprobe staining for $n k x 2.5$ transcripts at $12 \mathrm{hpf}$ in WT and hey2MO

751 embryos. Expanded expression of $n k x 2.5$ was observed in morphants (B) compared to

752 controls (A). (C-D) Confocal imaging of Mef2 in $T g(m y l 7: E G F P)$ control embryos

753 (C) and hey $2^{h s c 25}$ mutants (D). (E-F) Bar graph analysis displaying total number of

754 Mef2+ cells in the heart proper (E) and adjacent to the arterial pole ( $\mathrm{F}$, arrowheads)

755 between controls and mutants at $48 \mathrm{hpf}(\mathrm{N}=3, \mathrm{n}=4) .(\mathrm{G}-\mathrm{H})$ Confocal imaging of EdU

756 incorporation assay. Embryos were pulsed at $24 \mathrm{hpf}$ and imaged at $35 \mathrm{hpf}$. (I) Bar

757 graph showing the proliferative index of Edu+/nkx2.5+ cardiomyocytes between

758 controls and hey2 morphants when pulsed at $24 \mathrm{hpf}(\mathrm{N}=2, \mathrm{n}=4)$. No significant

759 difference in proliferation was observed. Error bars mean \pm sem; $* \mathrm{p}<0.05, * * \mathrm{p}<0.01$,

$760 * * * \mathrm{p}<0.001 ; \mathrm{n} . \mathrm{s}$, no significance. Scale bars $50 \mu \mathrm{m}$. 


\section{REFERENCES}

\section{5}

766

767

768

769

770

771

772

773

774

775

776

777

778
Abu-Issa, R., Waldo, K., and Kirby, M.L. (2004). Heart fields: one, two or more? Dev Biol 272, 281-285.

Alexander, J., Stainier, D.Y., and Yelon, D. (1998). Screening mosaic F1 females for mutations affecting zebrafish heart induction and patterning. Dev Genet 22, 288-299.

Auman, H.J., Coleman, H., Riley, H.E., Olale, F., Tsai, H.J., and Yelon, D. (2007). Functional modulation of cardiac form through regionally confined cell shape changes. PLoS Biol 5, e53.

Buckingham, M., Meilhac, S., and Zaffran, S. (2005). Building the mammalian heart from two sources of myocardial cells. Nat Rev Genet 6, 826-835.

Burg, L., Zhang, K., Bonawitz, T., Grajevskaja, V., Bellipanni, G., Waring, R., and Balciunas, D. (2016). Internal epitope tagging informed by relative lack of sequence conservation. Sci Rep 6, 36986.

Cai, C.L., Liang, X., Shi, Y., Chu, P.H., Pfaff, S.L., Chen, J., and Evans, S. (2003). Isl1 identifies a cardiac progenitor population that proliferates prior to differentiation and contributes a majority of cells to the heart. Dev Cell 5, 877-889.

Camp, E., Dietrich, S., and Munsterberg, A. (2012). Fate mapping identifies the origin of SHF/AHF progenitors in the chick primitive streak. PLoS ONE 7, e51948.

Chapman, D.L., Garvey, N., Hancock, S., Alexiou, M., Agulnik, S.I., Gibson-Brown, J.J., Cebra-Thomas, J., Bollag, R.J., Silver, L.M., and Papaioannou, V.E. (1996). Expression of the T-box family genes, Tbx1-Tbx5, during early mouse development. Dev Dyn 206, 379-390.

Chen, J.N., and Fishman, M.C. (1996). Zebrafish tinman homolog demarcates the heart field and initiates myocardial differentiation. Development 122, 3809-3816.

Choi, S.Y., Chacon-Heszele, M.F., Huang, L., McKenna, S., Wilson, F.P., Zuo, X., and Lipschutz, J.H. (2013). Cdc42 deficiency causes ciliary abnormalities and cystic kidneys. J Am Soc Nephrol 24, 1435-1450.

Davis, R.L., and Turner, D.L. (2001). Vertebrate hairy and Enhancer of split related proteins: transcriptional repressors regulating cellular differentiation and embryonic patterning. Oncogene 20, 8342-8357.

de Pater, E., Clijsters, L., Marques, S.R., Lin, Y.F., Garavito-Aguilar, Z.V., Yelon, D., and Bakkers, J. (2009). Distinct phases of cardiomyocyte differentiation regulate growth of the zebrafish heart. Development 136, 1633-1641.

Devine, W.P., Wythe, J.D., George, M., Koshiba-Takeuchi, K., and Bruneau, B.G. (2014). Early patterning and specification of cardiac progenitors in gastrulating mesoderm. eLife 3 , e03848. 
801 Doetzlhofer, A., Basch, M.L., Ohyama, T., Gessler, M., Groves, A.K., and Segil, N. 802 (2009). Hey2 regulation by FGF provides a Notch-independent mechanism for 803 maintaining pillar cell fate in the organ of Corti. Dev Cell 16, 58-69.

804 Donovan, J., Kordylewska, A., Jan, Y.N., and Utset, M.F. (2002). Tetralogy of fallot 805 and other congenital heart defects in Hey2 mutant mice. Curr Biol 12, 1605-1610.

806

807

808

809

810

811

812

813

814

815

816

817

818

819

820

821

822

823

824

825

826

827

828

829

830

831

832

833

834

835

836

837

838

Feng, L., Hernandez, R.E., Waxman, J.S., Yelon, D., and Moens, C.B. (2010). Dhrs3a regulates retinoic acid biosynthesis through a feedback inhibition mechanism. Dev Biol 338, 1-14.

Gerrard, D.T., Berry, A.A., Jennings, R.E., Piper Hanley, K., Bobola, N., and Hanley, N.A. (2016). An integrative transcriptomic atlas of organogenesis in human embryos. Elife 5, e15657.

Grimes, A.C., Stadt, H.A., Shepherd, I.T., and Kirby, M.L. (2006). Solving an enigma: arterial pole development in the zebrafish heart. Dev Biol 290, 265-276.

Guner-Ataman, B., Paffett-Lugassy, N., Adams, M.S., Nevis, K.R., Jahangiri, L., Obregon, P., Kikuchi, K., Poss, K.D., Burns, C.E., and Burns, C.G. (2013). Zebrafish second heart field development relies on progenitor specification in anterior lateral plate mesoderm and nkx2.5 function. Development 140, 1353-1363.

Hami, D., Grimes, A.C., Tsai, H.J., and Kirby, M.L. (2011). Zebrafish cardiac development requires a conserved secondary heart field. Development 138, 23892398.

Hermkens, D.M., van Impel, A., Urasaki, A., Bussmann, J., Duckers, H.J., and Schulte-Merker, S. (2015). Sox7 controls arterial specification in conjunction with hey2 and efnb2 function. Development 142, 1695-1704.

Huang, C.J., Tu, C.T., Hsiao, C.D., Hsieh, F.J., and Tsai, H.J. (2003). Germ-line transmission of a myocardium-specific GFP transgene reveals critical regulatory elements in the cardiac myosin light chain 2 promoter of zebrafish. Dev Dyn 228, 3040 .

Hutson, M.R., Zeng, X.L., Kim, A.J., Antoon, E., Harward, S., and Kirby, M.L. (2010). Arterial pole progenitors interpret opposing FGF/BMP signals to proliferate or differentiate. Development 137, 3001-3011.

Ilagan, R., Abu-Issa, R., Brown, D., Yang, Y.P., Jiao, K., Schwartz, R.J., Klingensmith, J., and Meyers, E.N. (2006). Fgf8 is required for anterior heart field development. Development 133, 2435-2445.

Ivanovitch, K., Temino, S., and Torres, M. (2017). Live imaging of heart tube development in mouse reveals alternating phases of cardiac differentiation and morphogenesis. bioRxiv 10.1101/170522.

Jahangiri, L., Sharpe, M., Novikov, N., Gonzalez-Rosa, J.M., Borikova, A., Nevis, K., Paffett-Lugassy, N., Zhao, L., Adams, M., Guner-Ataman, B., et al. (2016). The AP-1 
transcription factor component Fosl2 potentiates the rate of myocardial differentiation from the zebrafish second heart field. Development 143, 113-122.

841 Jia, H., King, I.N., Chopra, S.S., Wan, H., Ni, T.T., Jiang, C., Guan, X., Wells, S., 842 Srivastava, D., and Zhong, T.P. (2007). Vertebrate heart growth is regulated by 843 functional antagonism between Gridlock and Gata5. Proc Natl Acad Sci U S A 104, 844 14008-14013.

845 Kelly, R.G. (2012). The second heart field. Curr Top Dev Biol 100, 33-65.

846 Kikuchi, K., Holdway, J.E., Major, R.J., Blum, N., Dahn, R.D., Begemann, G., and 847 Poss, K.D. (2011). Retinoic acid production by endocardium and epicardium is an 848 injury response essential for zebrafish heart regeneration. Dev Cell 20, 397-404.

849 Kok, F.O., Shin, M., Ni, C.W., Gupta, A., Grosse, A.S., van Impel, A., Kirchmaier, 850 B.C., Peterson-Maduro, J., Kourkoulis, G., Male, I., et al. (2015). Reverse genetic 851 screening reveals poor correlation between morpholino-induced and mutant 852 phenotypes in zebrafish. Dev Cell 32, 97-108.

853 Kokubo, H., Tomita-Miyagawa, S., Hamada, Y., and Saga, Y. (2007). Hesr1 and 854 Hesr2 regulate atrioventricular boundary formation in the developing heart through 855 the repression of Tbx2. Development 134, 747-755.

856 Lazic, S., and Scott, I.C. (2011). Mef2cb regulates late myocardial cell addition from 857 a second heart field-like population of progenitors in zebrafish. Dev Biol 354, 123858133.

859 Lescroart, F., Kelly, R.G., Le Garrec, J.F., Nicolas, J.F., Meilhac, S.M., and 860 Buckingham, M. (2010). Clonal analysis reveals common lineage relationships 861 between head muscles and second heart field derivatives in the mouse embryo. 862 Development 137, 3269-3279.

863 Li, D., Sinha, T., Ajima, R., Seo, H.S., Yamaguchi, T.P., and Wang, J. (2016). Spatial 864 regulation of cell cohesion by Wnt5a during second heart field progenitor 865 deployment. Dev Biol 412, 18-31.

866 Li, Q., Ritter, D., Yang, N., Dong, Z., Li, H., Chuang, J.H., and Guo, S. (2010). A 867 systematic approach to identify functional motifs within vertebrate developmental 868 enhancers. Dev Biol 337, 484-495.

869 Lou, X., Deshwar, A.R., Crump, J.G., and Scott, I.C. (2011). Smarcd3b and Gata5 870 promote a cardiac progenitor fate in the zebrafish embryo. Development 138, 31138713123.

872 Ma, L., Lu, M.F., Schwartz, R.J., and Martin, J.F. (2005). Bmp2 is essential for 873 cardiac cushion epithelial-mesenchymal transition and myocardial patterning. 874 Development 132, 5601-5611.

875 Manasek, F.J. (1981). Determinants of heart shape in early embryos. Federation 876 proceedings 40, 2011-2016. 
Manasek, F.J., Burnside, M.B., and Waterman, R.E. (1972). Myocardial cell shape change as a mechanism of embryonic heart looping. Dev Biol 29, 349-371.

Mandal, A., Holowiecki, A., Song, Y.C., and Waxman, J.S. (2017). Wnt signaling balances specification of the cardiac and pharyngeal muscle fields. Mech Dev 143, $32-41$.

Meilhac, S.M., Kelly, R.G., Rocancourt, D., Eloy-Trinquet, S., Nicolas, J.F., and Buckingham, M.E. (2003). A retrospective clonal analysis of the myocardium reveals two phases of clonal growth in the developing mouse heart. Development 130, 38773889 .

Mjaatvedt, C.H., Nakaoka, T., Moreno-Rodriguez, R., Norris, R.A., Kern, M.J., Eisenberg, C.A., Turner, D., and Markwald, R.R. (2001). The outflow tract of the heart is recruited from a novel heart-forming field. Dev Biol 238, 97-109.

Moorman, A.F., Christoffels, V.M., Anderson, R.H., and van den Hoff, M.J. (2007). The heart-forming fields: one or multiple? Philos Trans R Soc Lond B Biol Sci 362, 1257-1265.

Nevis, K., Obregon, P., Walsh, C., Guner-Ataman, B., Burns, C.G., and Burns, C.E. (2013). Tbx1 is required for second heart field proliferation in zebrafish. Dev Dyn 242, 550-559.

Paffett-Lugassy, N., Singh, R., Nevis, K.R., Guner-Ataman, B., O'Loughlin, E., Jahangiri, L., Harvey, R.P., Burns, C.G., and Burns, C.E. (2013). Heart field origin of great vessel precursors relies on nkx2.5-mediated vasculogenesis. Nat Cell Biol 15, 1362-1369.

Park, E.J., Watanabe, Y., Smyth, G., Miyagawa-Tomita, S., Meyers, E., Klingensmith, J., Camenisch, T., Buckingham, M., and Moon, A.M. (2008). An FGF autocrine loop initiated in second heart field mesoderm regulates morphogenesis at the arterial pole of the heart. Development 135, 3599-3610.

Prall, O.W., Menon, M.K., Solloway, M.J., Watanabe, Y., Zaffran, S., Bajolle, F., Biben, C., McBride, J.J., Robertson, B.R., Chaulet, H., et al. (2007). An nkx25/bmp2/smad1 negative feedback loop controls heart progenitor specification and proliferation. Cell 128, 947-959.

Rochais, F., Mesbah, K., and Kelly, R.G. (2009). Signaling pathways controlling second heart field development. Circ Res 104, 933-942.

Rutenberg, J.B., Fischer, A., Jia, H., Gessler, M., Zhong, T.P., and Mercola, M. (2006). Developmental patterning of the cardiac atrioventricular canal by Notch and Hairy-related transcription factors. Development 133, 4381-4390.

Ryckebusch, L., Wang, Z., Bertrand, N., Lin, S.C., Chi, X., Schwartz, R., Zaffran, S., and Niederreither, K. (2008). Retinoic acid deficiency alters second heart field formation. Proc Natl Acad Sci U S A 105, 2913-2918. 
915 Sakata, Y., Kamei, C.N., Nakagami, H., Bronson, R., Liao, J.K., and Chin, M.T. 916 (2002). Ventricular septal defect and cardiomyopathy in mice lacking the 917 transcription factor CHF1/Hey2. Proc Natl Acad Sci U S A 99, 16197-16202.

918 Scott, I.C., Masri, B., D'Amico, L.A., Jin, S.W., Jungblut, B., Wehman, A., Baier, H., 919 Audigier, Y., and Stainier, D.Y.R. (2007). The G-Protein Coupled Receptor Agtrl1b 920 Regulates Early Development of Myocardial Progenitors. Developmental Cell 12, 921 403-413.

922

923

924

925

926

927

928

929

930 931

932 933

Sirbu, I.O., Zhao, X., and Duester, G. (2008). Retinoic acid controls heart anteroposterior patterning by down-regulating Isl1 through the Fgf8 pathway. Dev Dyn 237, 1627-1635.

Sorrell, M.R., and Waxman, J.S. (2011). Restraint of Fgf8 signaling by retinoic acid signaling is required for proper heart and forelimb formation. Dev Biol 358, 44-55.

Stainier, D.Y., Lee, R.K., and Fishman, M.C. (1993). Cardiovascular development in the zebrafish. I. Myocardial fate map and heart tube formation. Development 119, 3140 .

Taber, L.A. (2006). Biophysical mechanisms of cardiac looping. Int J Dev Biol 50, 323-332.

Tang, R., Dodd, A., Lai, D., McNabb, W.C., and Love, D.R. (2007). Validation of zebrafish (Danio rerio) reference genes for quantitative real-time RT-PCR normalization. Acta biochimica et biophysica Sinica 39, 384-390.

Thisse, C., and Thisse, B. (2008). High-resolution in situ hybridization to wholemount zebrafish embryos. Nat Protoc 3, 59-69.

Tirosh-Finkel, L., Zeisel, A., Brodt-Ivenshitz, M., Shamai, A., Yao, Z., Seger, R., Domany, E., and Tzahor, E. (2010). BMP-mediated inhibition of FGF signaling promotes cardiomyocyte differentiation of anterior heart field progenitors. Development 137, 2989-3000.

Traver, D., Paw, B.H., Poss, K.D., Penberthy, W.T., Lin, S., and Zon, L.I. (2003). Transplantation and in vivo imaging of multilineage engraftment in zebrafish bloodless mutants. Nat Immunol 4, 1238-1246.

van den Berg, G., Abu-Issa, R., de Boer, B.A., Hutson, M.R., de Boer, P.A., Soufan, A.T., Ruijter, J.M., Kirby, M.L., van den Hoff, M.J., and Moorman, A.F. (2009). A caudal proliferating growth center contributes to both poles of the forming heart tube. Circ Res 104, 179-188.

Waldo, K.L., Hutson, M.R., Ward, C.C., Zdanowicz, M., Stadt, H.A., Kumiski, D., Abu-Issa, R., and Kirby, M.L. (2005). Secondary heart field contributes myocardium and smooth muscle to the arterial pole of the developing heart. Dev Biol 281, 78-90.

Ward, C., Stadt, H., Hutson, M., and Kirby, M.L. (2005). Ablation of the secondary heart field leads to tetralogy of Fallot and pulmonary atresia. Dev Biol 284, 72-83. 
953 Waxman, J.S., Keegan, B.R., Roberts, R.W., Poss, K.D., and Yelon, D. (2008).

954 Hoxb5b acts downstream of retinoic acid signaling in the forelimb field to restrict 955 heart field potential in zebrafish. Dev Cell 15, 923-934.

956 Weinstein, B.M., Stemple, D.L., Driever, W., and Fishman, M.C. (1995). Gridlock, a 957 localized heritable vascular patterning defect in the zebrafish. Nature medicine 1 , 958 1143-1147.

959 Westerfield, M. (1993). The Zebrafish Book: A Guide for the Laboratory Use of 960 Zebrafish Danio (Brachydanio) rerio., 2nd edition edn (Oregon: University of Oregon 961 Press).

962 Winkler, C., Elmasri, H., Klamt, B., Volff, J.N., and Gessler, M. (2003). 963 Characterization of hey bHLH genes in teleost fish. Dev Genes Evol 213, 541-553.

964 Witzel, H.R., Cheedipudi, S., Gao, R., Stainier, D.Y., and Dobreva, G.D. (2017). 965 Isl2b regulates anterior second heart field development in zebrafish. Sci Rep 7, 41043.

966 Witzel, H.R., Jungblut, B., Choe, C.P., Crump, J.G., Braun, T., and Dobreva, G. 967 (2012). The LIM protein Ajuba restricts the second heart field progenitor pool by 968 regulating Isl1 activity. Dev Cell 23, 58-70.

969 Yelon, D., Horne, S.A., and Stainier, D.Y. (1999). Restricted expression of cardiac 970 myosin genes reveals regulated aspects of heart tube assembly in zebrafish. Dev Biol $971214,23-37$.

972 Yoruk, B., Gillers, B.S., Chi, N.C., and Scott, I.C. (2012). Ccm3 functions in a 973 manner distinct from $\mathrm{Ccm} 1$ and $\mathrm{Ccm} 2$ in a zebrafish model of CCM vascular disease. 974 Dev Biol 362, 121-131.

975 Zeng, X.X., and Yelon, D. (2014). Cadm4 restricts the production of cardiac outflow 976 tract progenitor cells. Cell Rep 7, 951-960.

977 Zhang, H., and Bradley, A. (1996). Mice deficient for BMP2 are nonviable and have 978 defects in amnion/chorion and cardiac development. Development 122, 2977-2986.

979 Zhang, L., Zhong, T., Wang, Y., Jiang, Q., Song, H., and Gui, Y. (2006). TBX1, a 980 DiGeorge syndrome candidate gene, is inhibited by retinoic acid. Int J Dev Biol 50, 981 55-61.

982 Zhao, C., Guo, H., Li, J., Myint, T., Pittman, W., Yang, L., Zhong, W., Schwartz, 983 R.J., Schwarz, J.J., Singer, H.A., et al. (2014). Numb family proteins are essential for 984 cardiac morphogenesis and progenitor differentiation. Development 141, 281-295.

985
Zhong, T.P., Childs, S., Leu, J.P., and Fishman, M.C. (2001). Gridlock signalling pathway fashions the first embryonic artery. Nature 414, 216-220.

Zhong, T.P., Rosenberg, M., Mohideen, M.A., Weinstein, B., and Fishman, M.C. (2000). gridlock, an HLH gene required for assembly of the aorta in zebrafish. Science 287, 1820-1824. 

protein 3 identifies a second heart field in zebrafish. Nature 474, 645-648. 
Figure 1

A

hey2/nkx2.5

15hpf
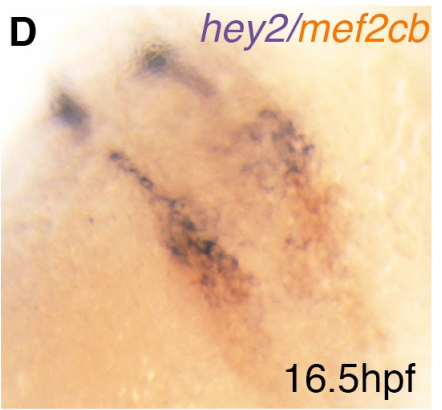

H GFP/my/7

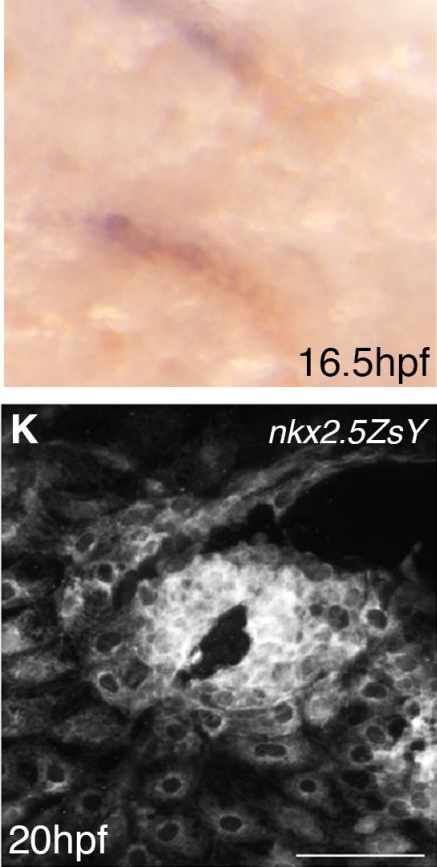

L

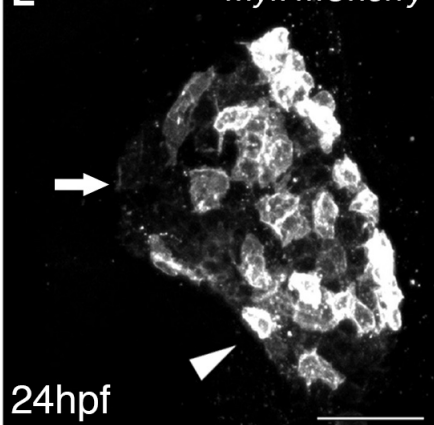

B

hey2/myl7

C

hey2/myl7

G

ALPM 16.5hpf

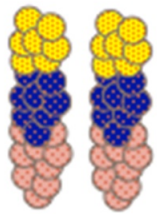

heart cone $22 \mathrm{hpf}$

24hpf

E

20hpf

hey2/mef2cb

F

hey2/mef2cb

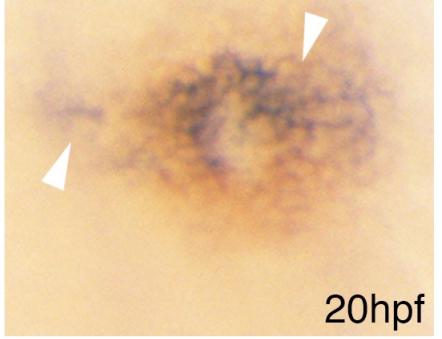

\section{I}

GFP/myl7 J 24hpf
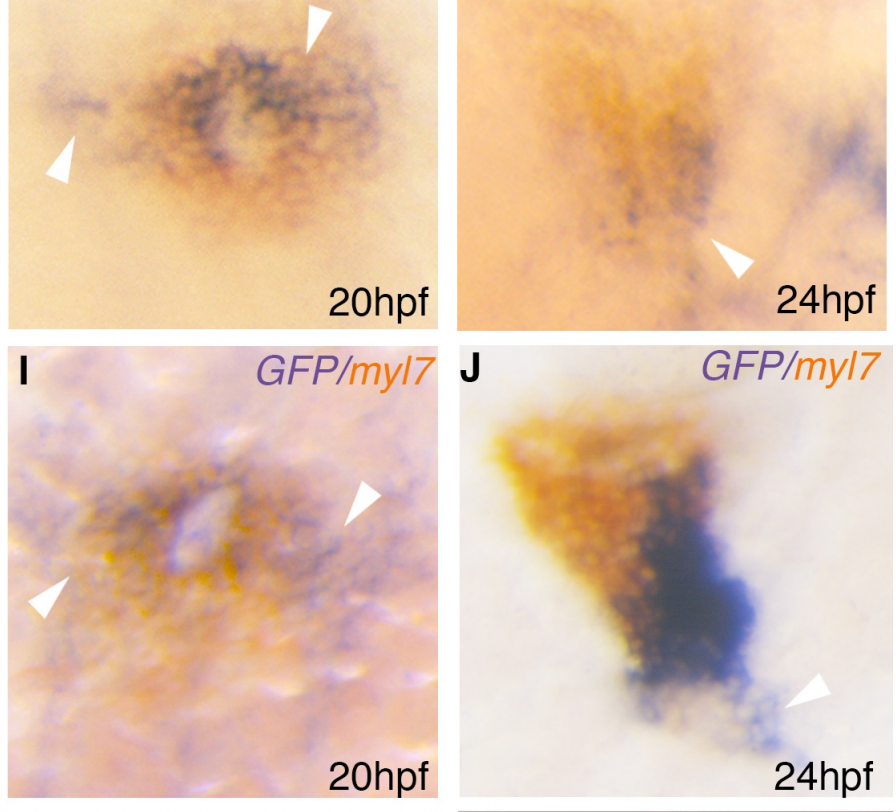

A

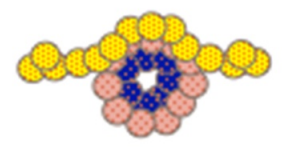

heart tube $24 \mathrm{hpf}$

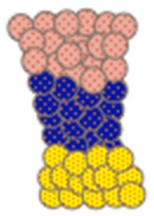

SHF

- FHF/SHF

FHF
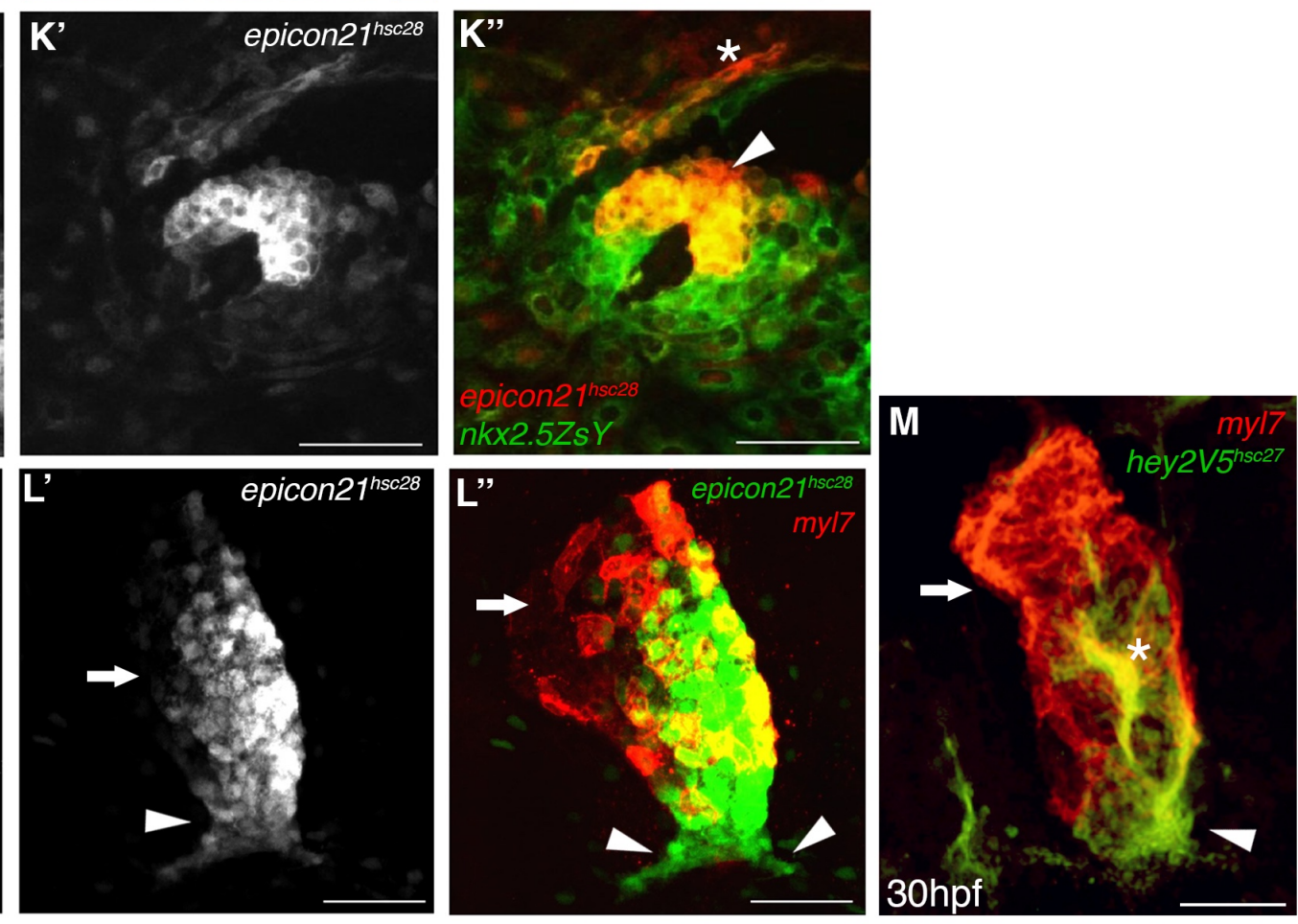
Figure. 2

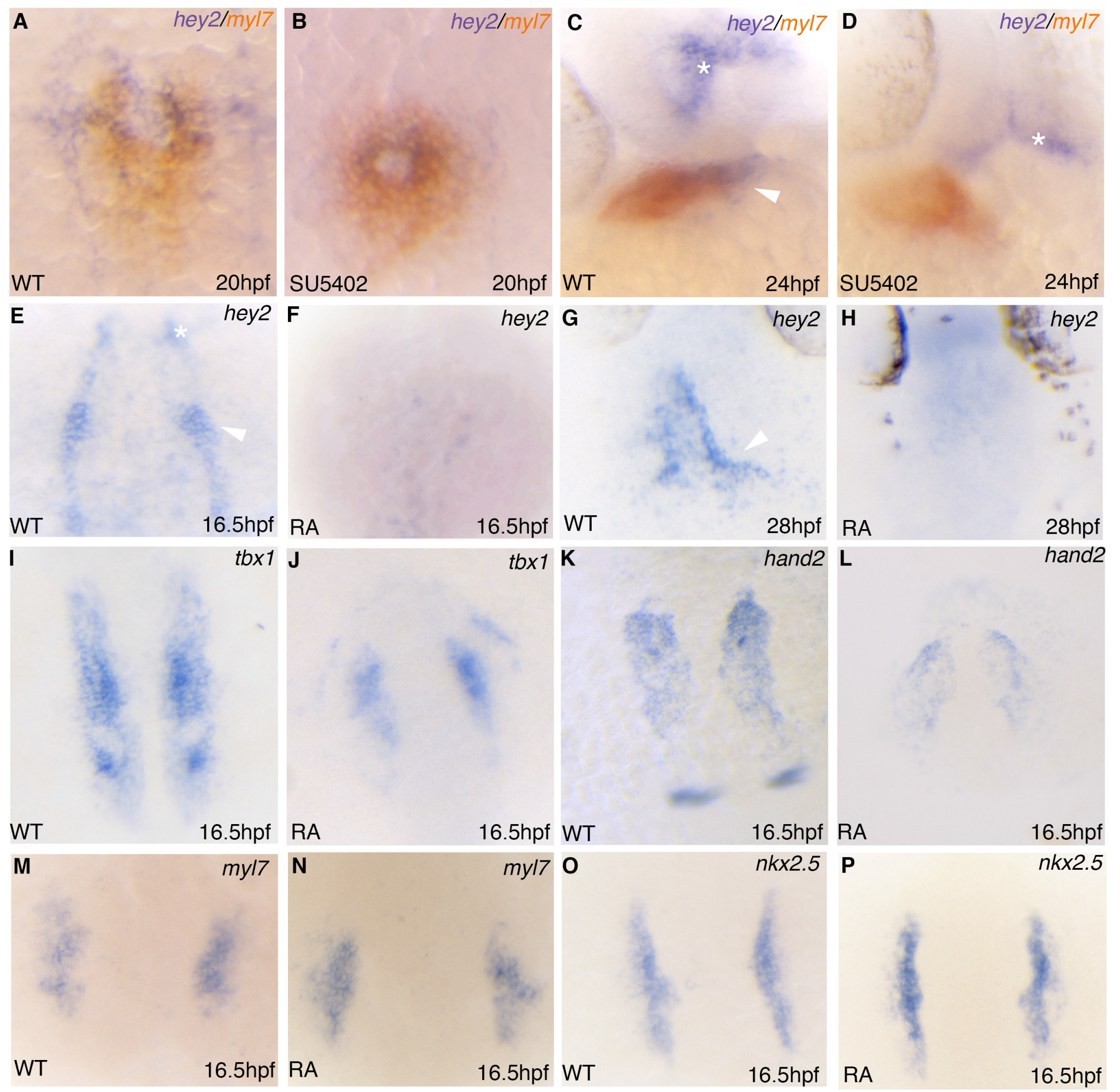


Figure 3

A
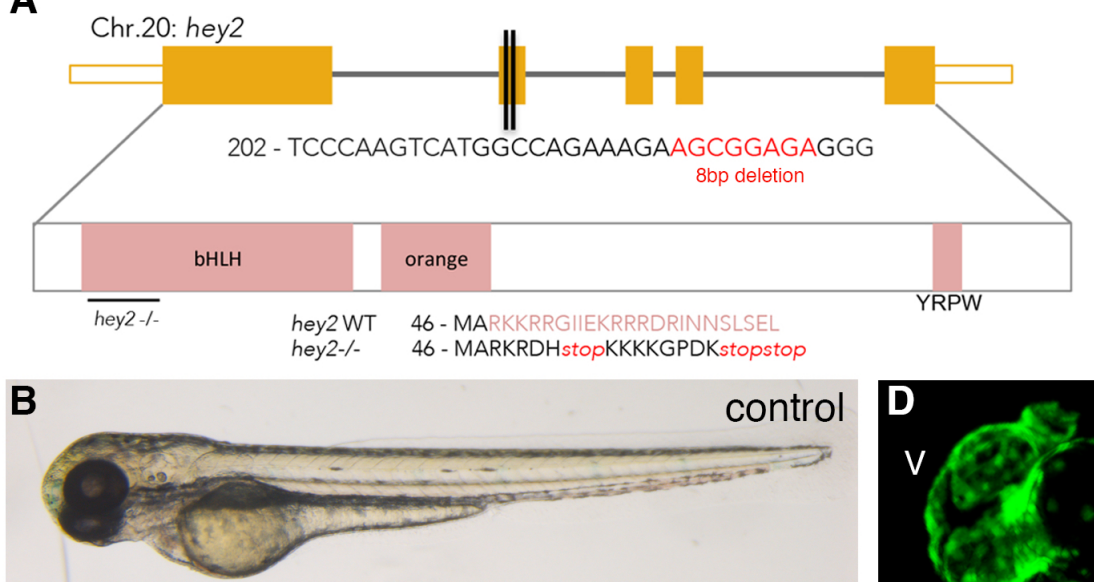

\section{$72 \mathrm{hpf}$}

C

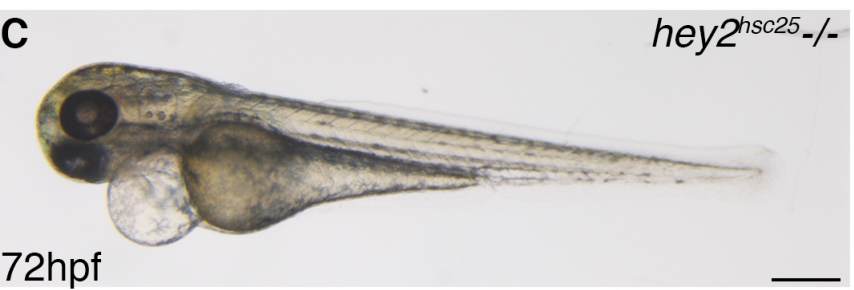

$72 \mathrm{hpf}$

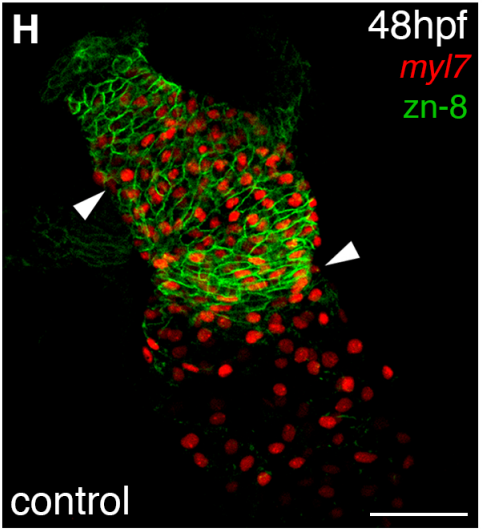

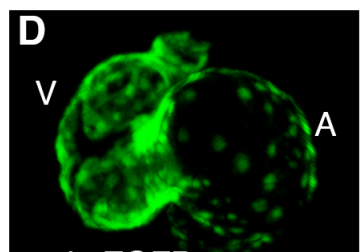

myl7:EGFP

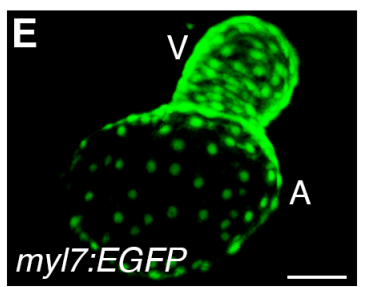

48hpf J

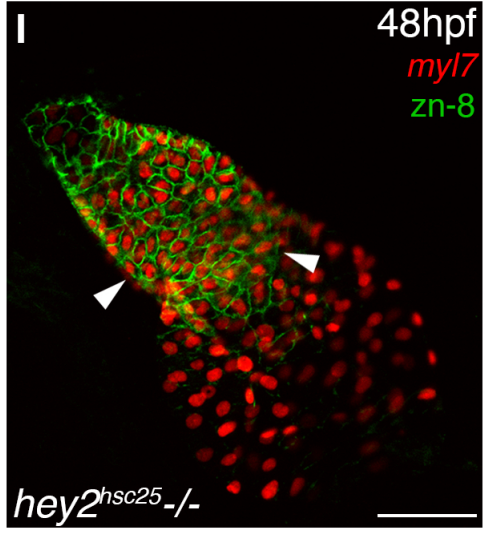

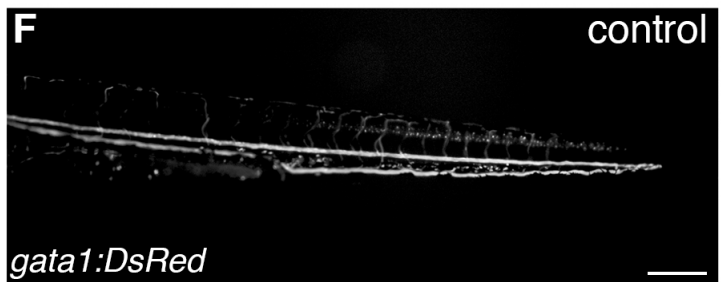
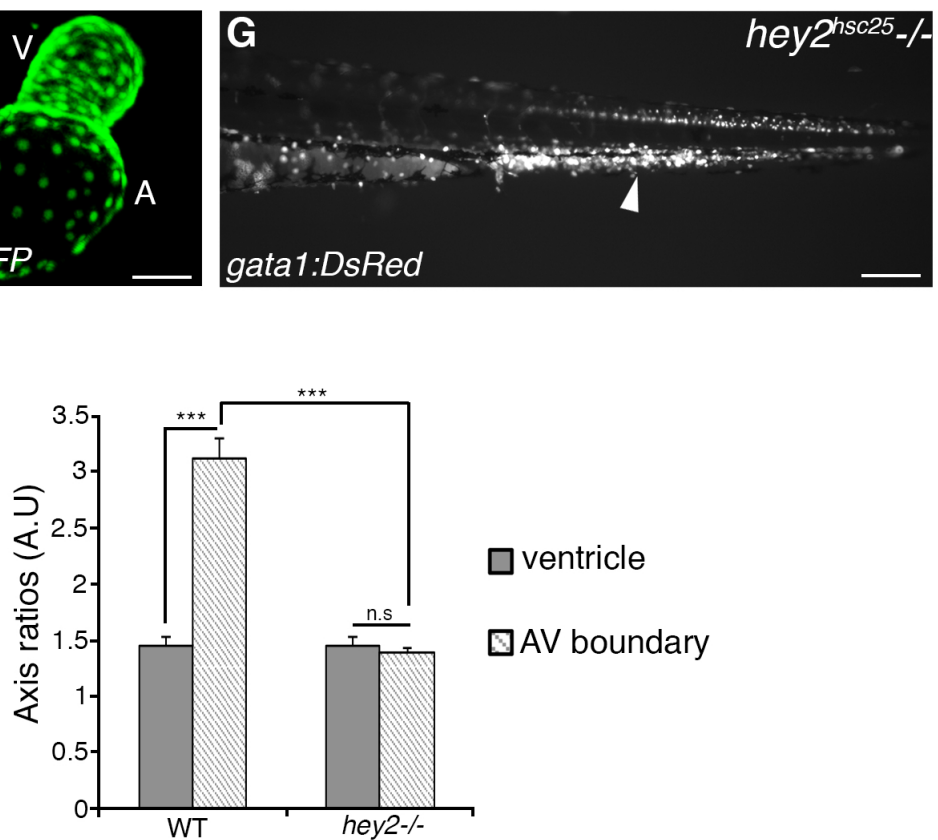
Figure 4
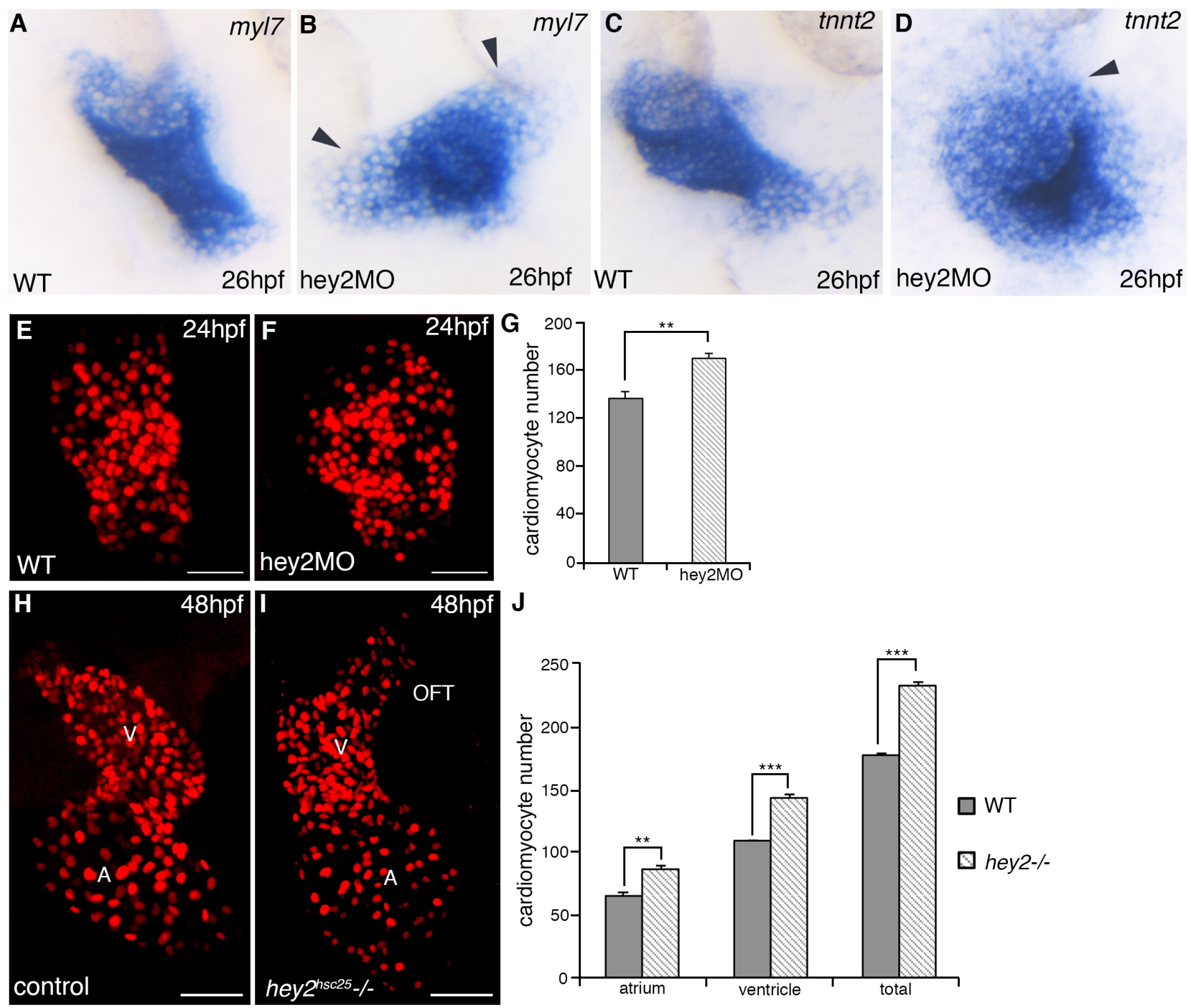
Figure 5

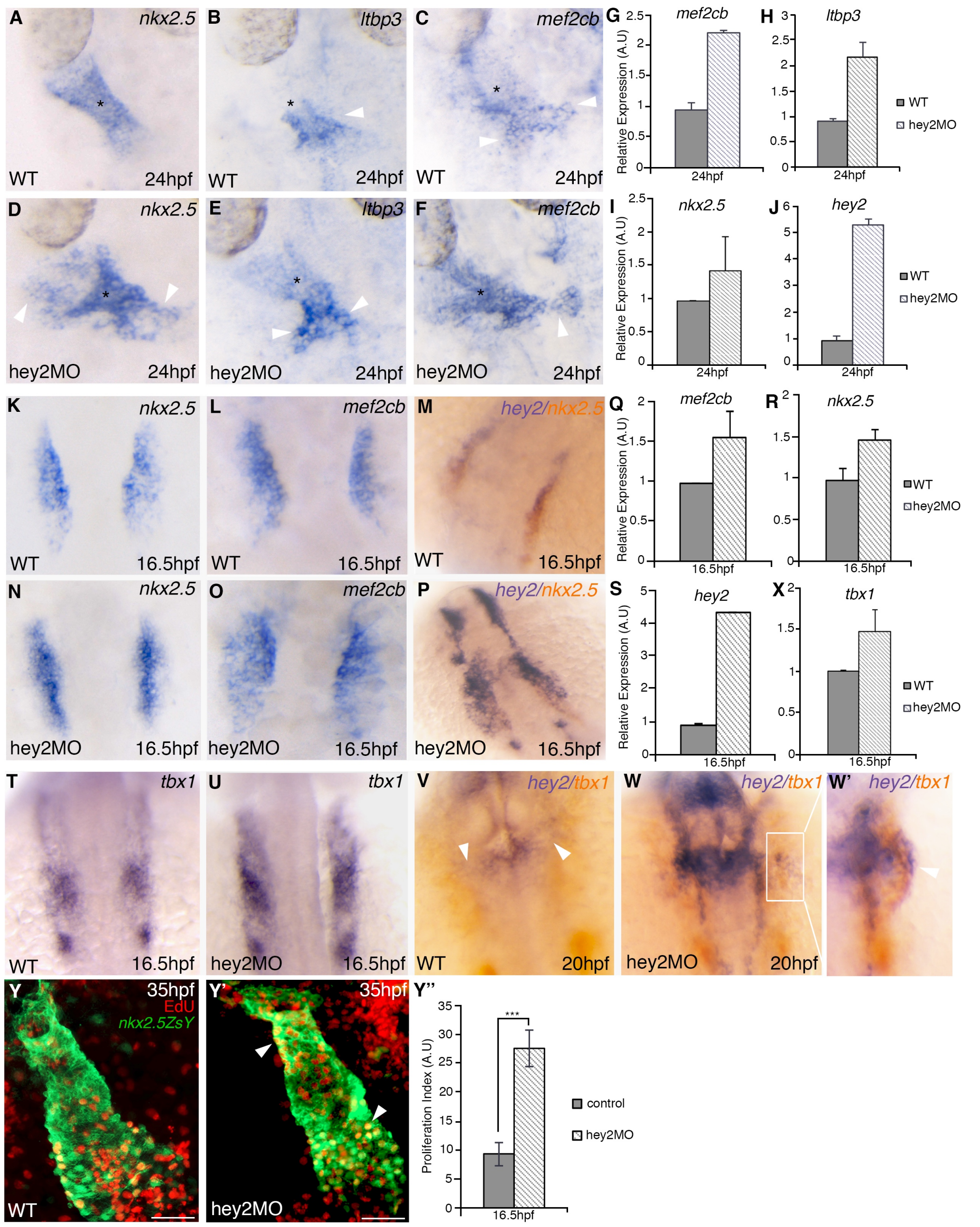


Figure 6

A

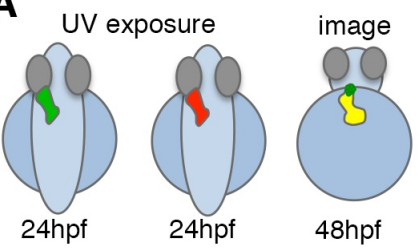

E

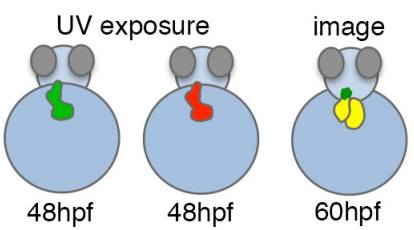

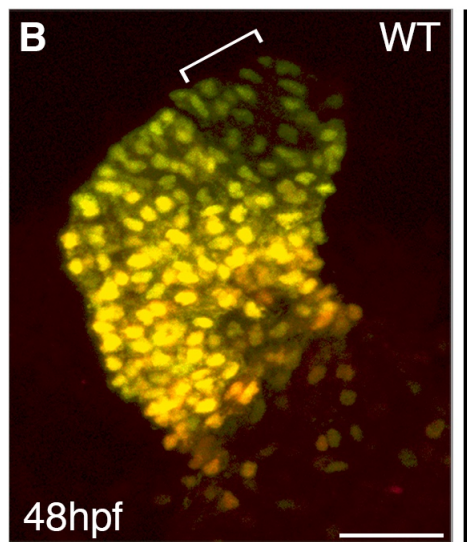
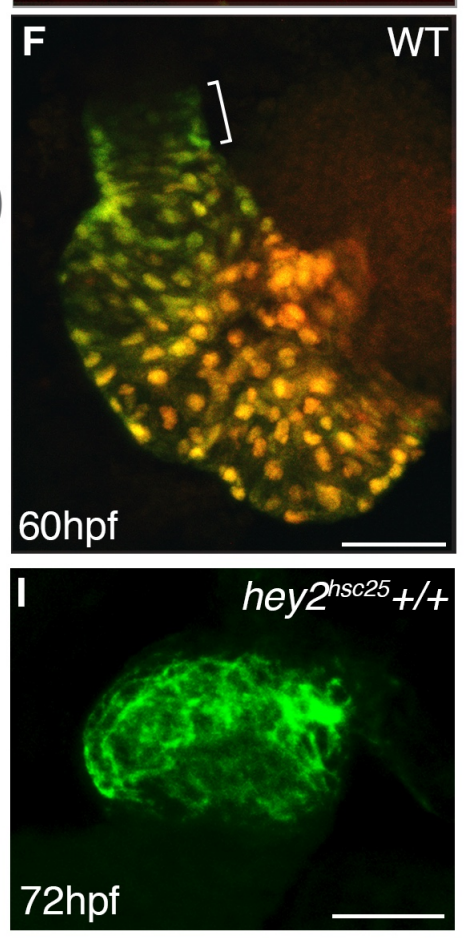
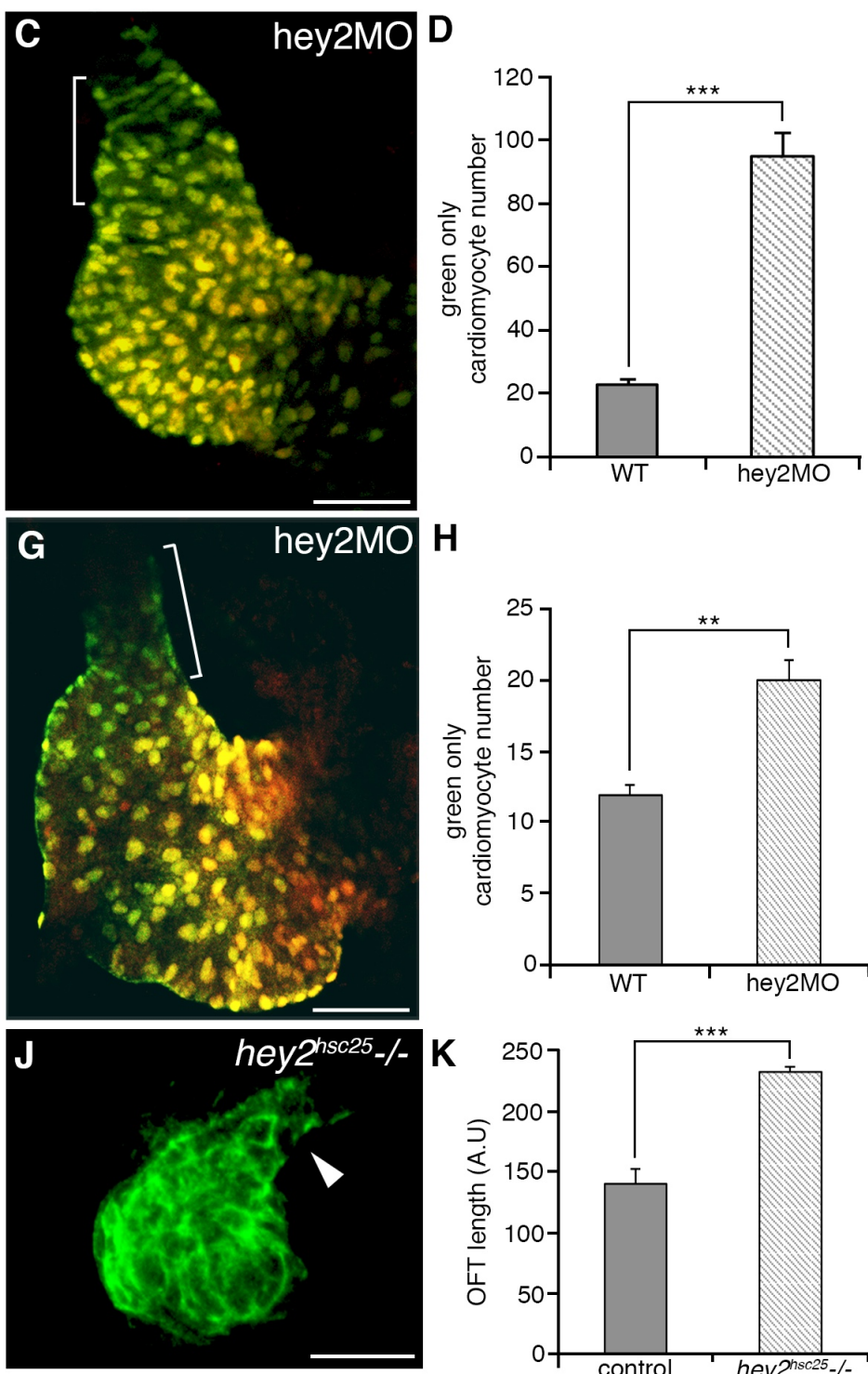

$\mathrm{H}$
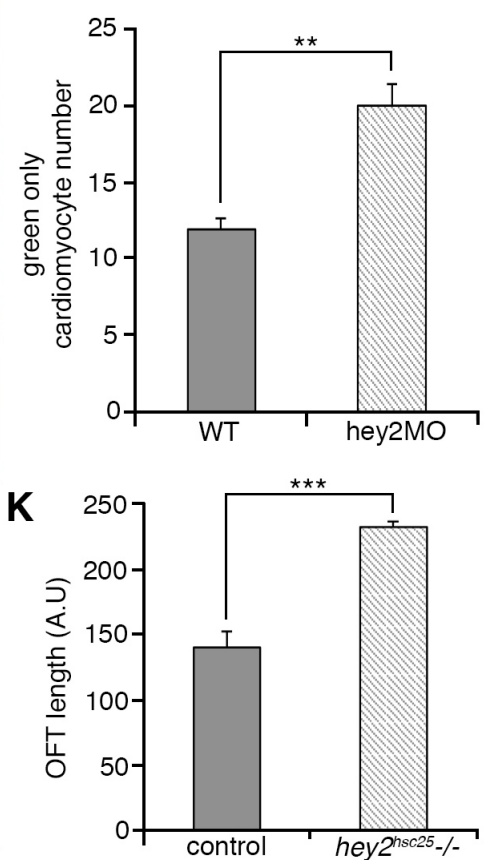
Figure. 7

A

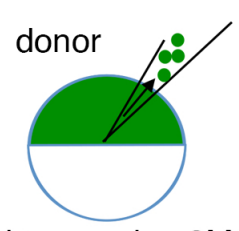

wild type or hey2MO

Tg(myl7:nlsKikGR)

B

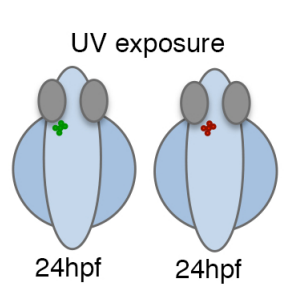

48hpf

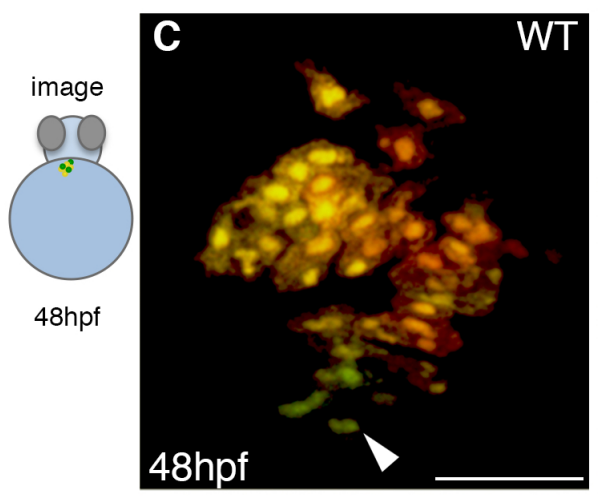

F
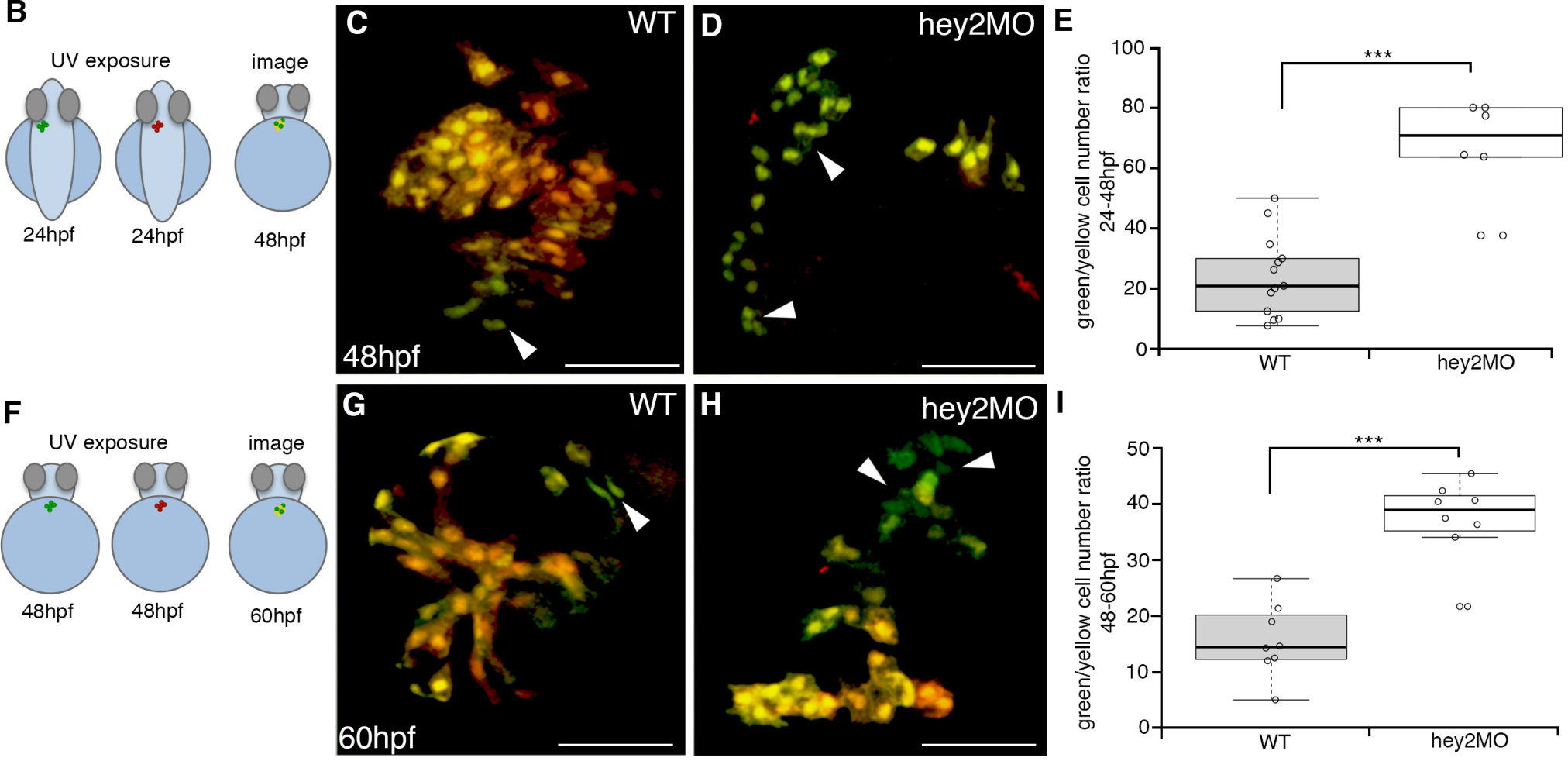

J
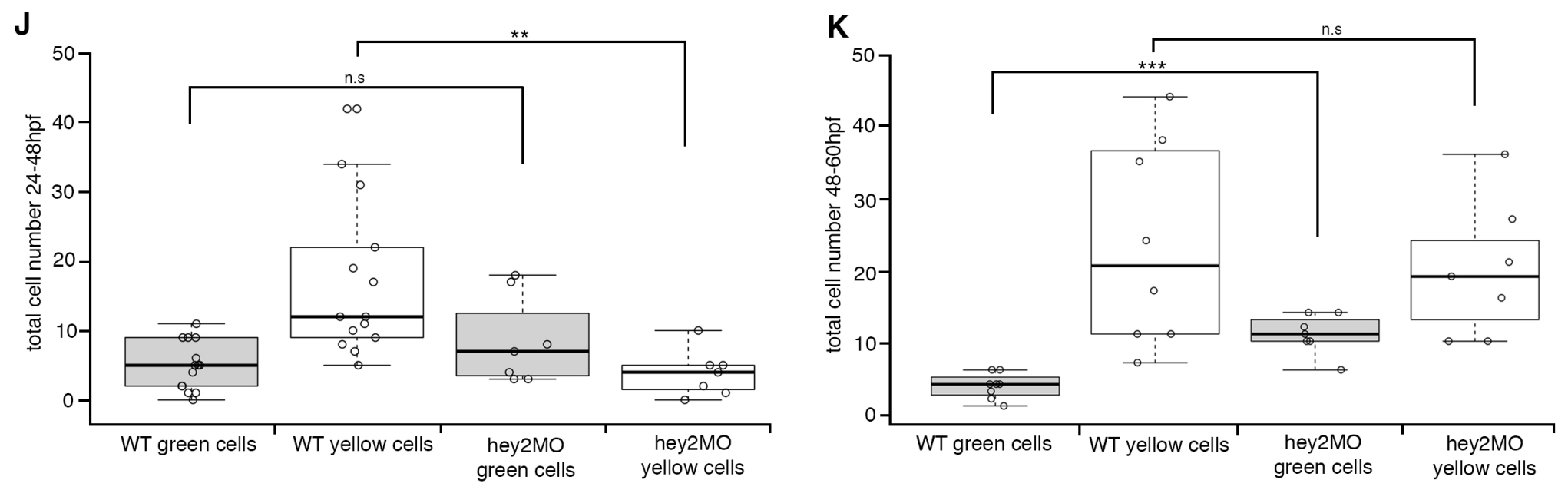
supplemental Fig.1
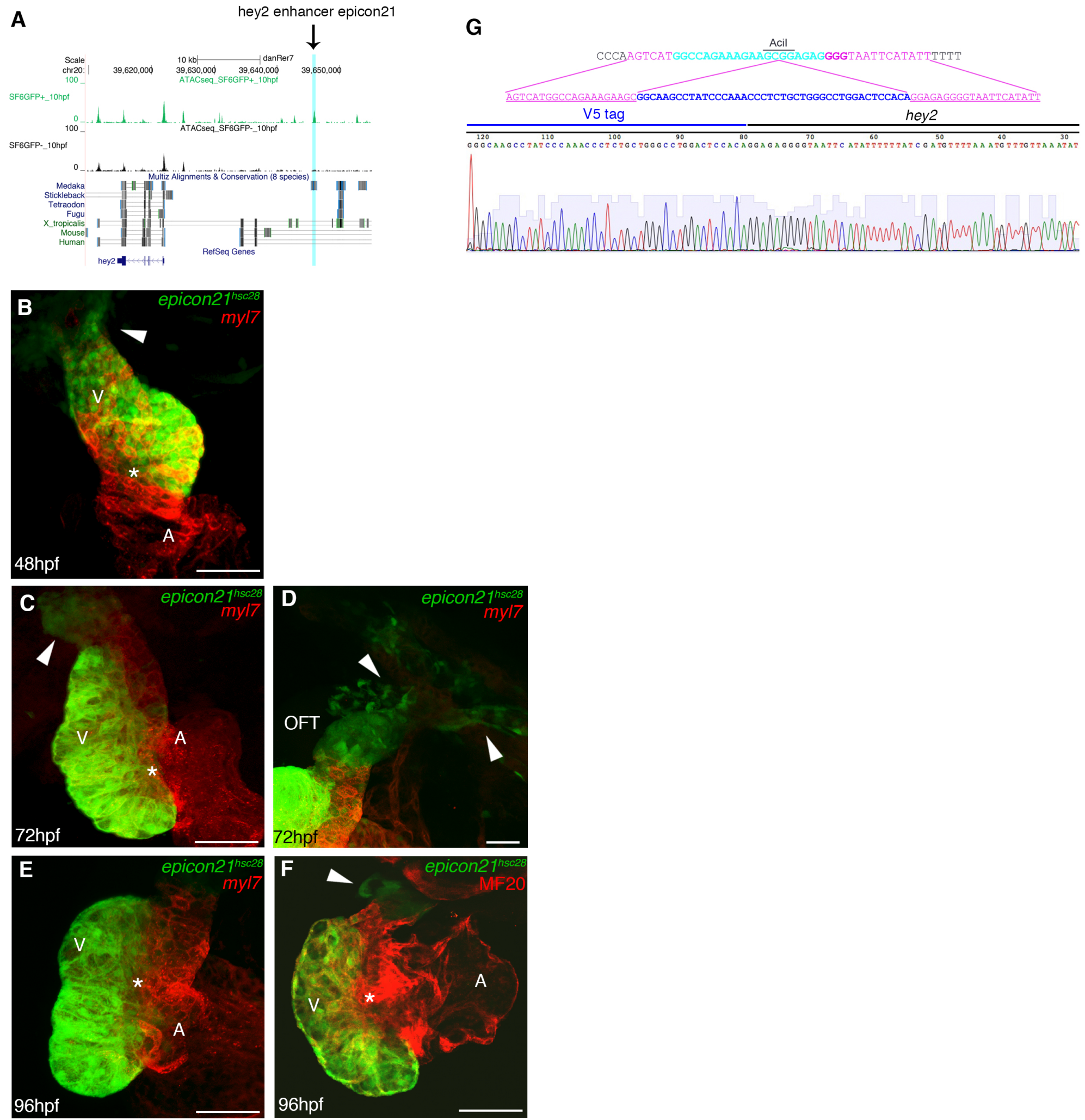
supplemental Fig.2

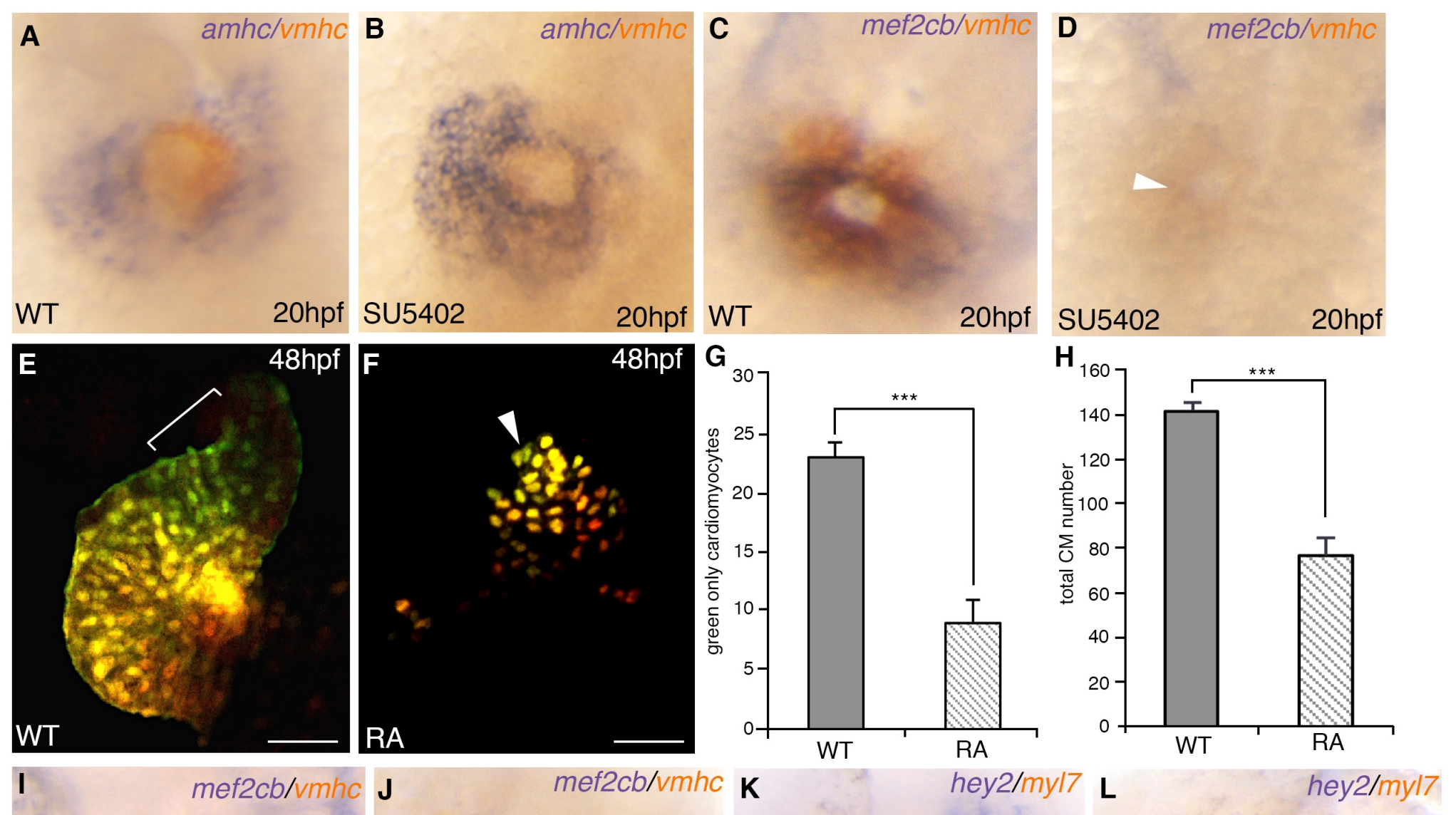

WT

28hpf RA

28hpf WT

28hpf RA

28hpf

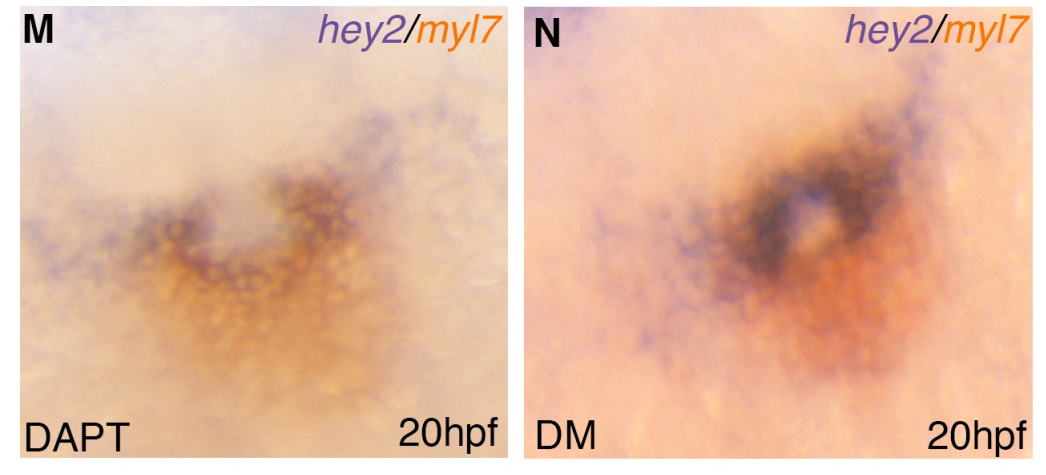


supplemental fig. 3
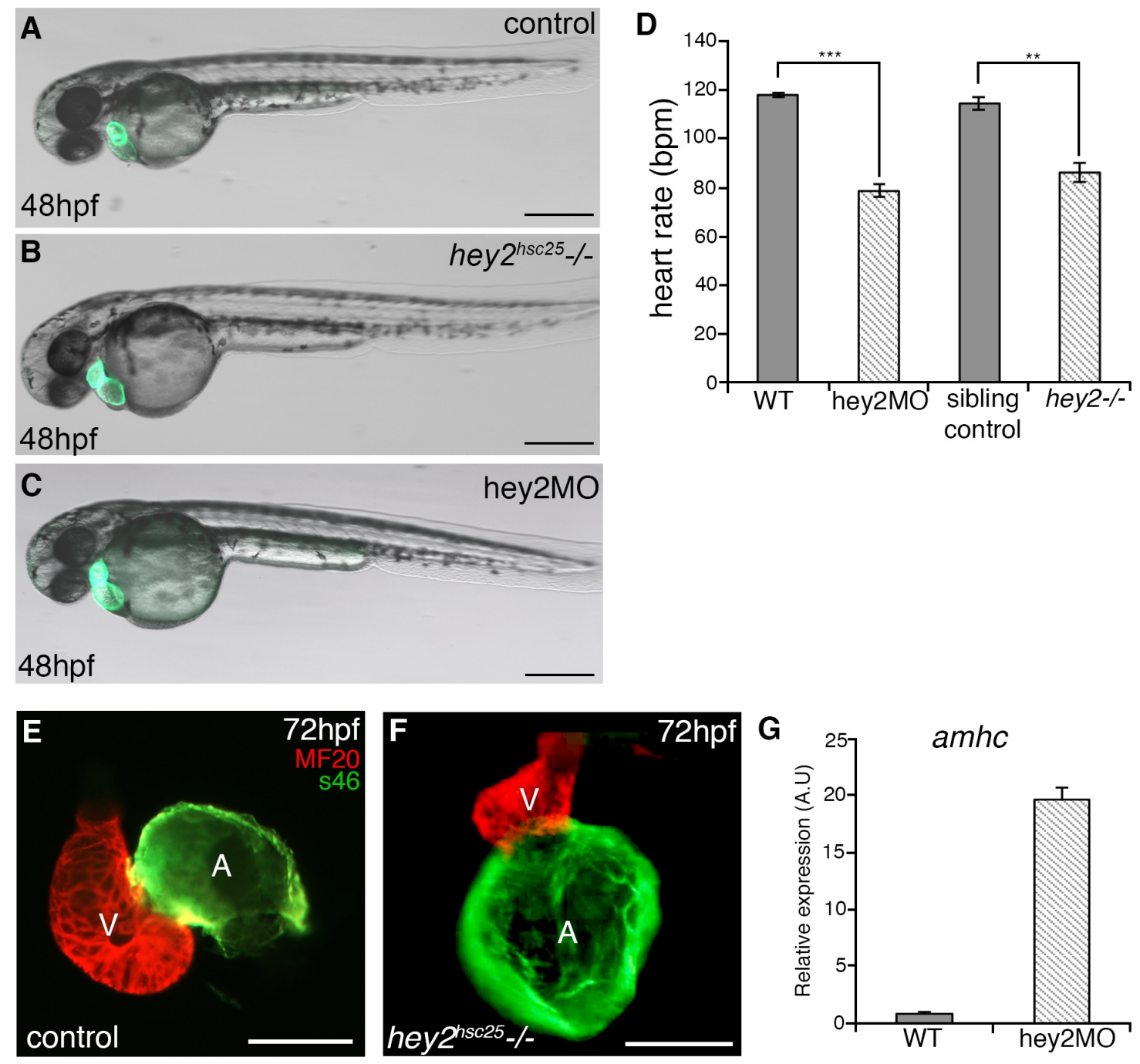

H

bmp4 I

$t b \times 2 b \quad \mathbf{L}$

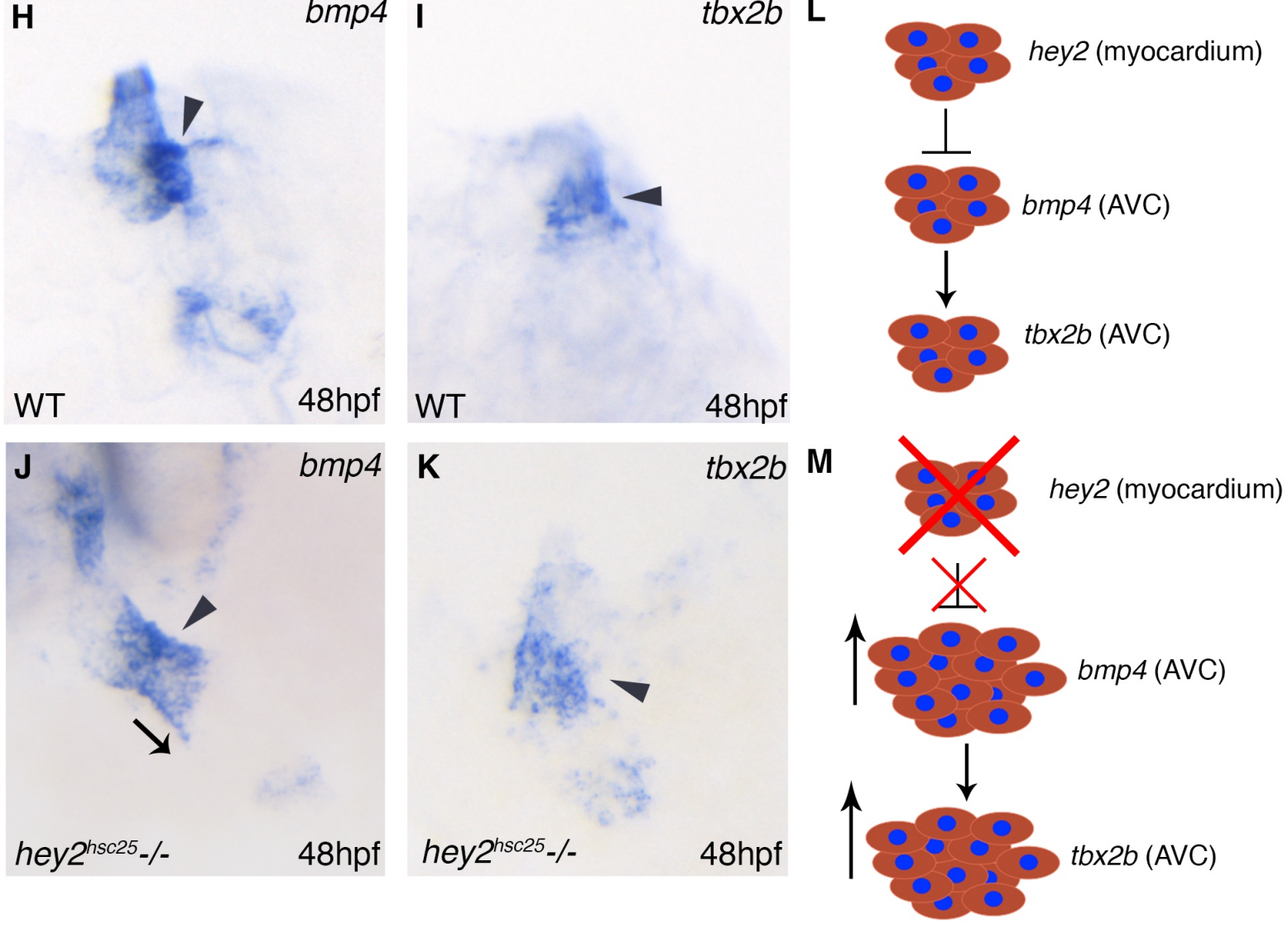


supplemental Fig.4

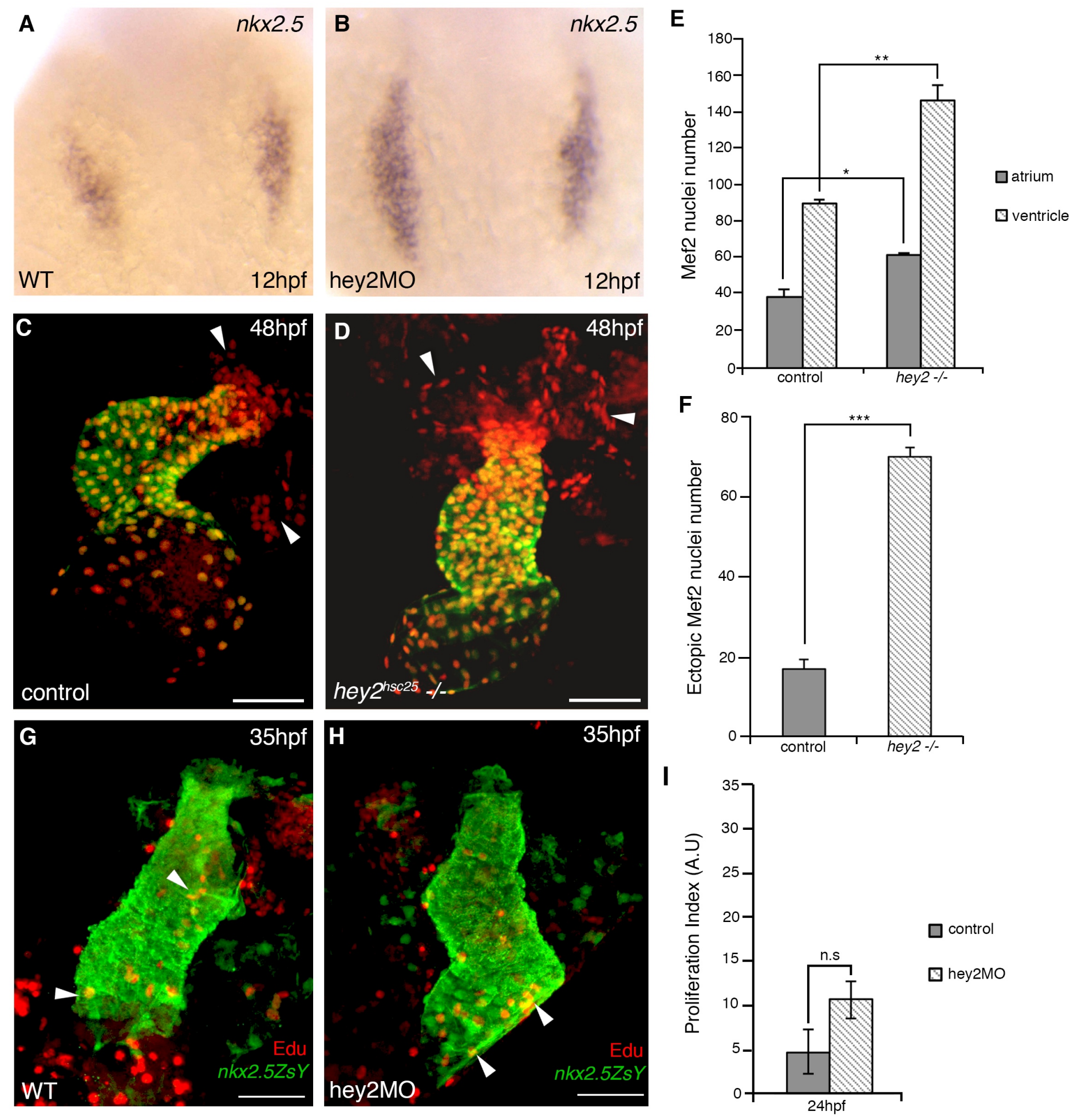

